\title{
Dark matter and collider signatures of the MSSM
}

\author{
Andrew Fowlie, ${ }^{1}$ Kamila Kowalska, ${ }^{2}$ Leszek Roszkowski, ${ }^{2 *}$ \\ Enrico Maria Sessolo, ${ }^{2}$ and Yue-Lin Sming Tsai ${ }^{2}$ \\ BayesFITS Group \\ ${ }^{1}$ Department of Physics and Astronomy, University of Sheffield, Sheffield S3 7RH, England \\ ${ }^{2}$ National Centre for Nuclear Research, Hoża 69, 00-681 Warsaw, Poland \\ a.fowlie@sheffield.ac.uk, Kamila.Kowalska@fuw.edu.pl \\ L.Roszkowski@sheffield.ac.uk, Enrico-Maria.Sessolo@fuw.edu.pl, \\ Sming. Tsai@fuw.edu.pl
}

\begin{abstract}
We explore the MSSM with 9 free parameters (p9MSSM) that have been selected as a minimum set that allows an investigation of neutralino dark matter and collider signatures while maintaining consistency with several constraints. These include measurement of the dark matter relic density from PLANCK, main properties of the discovered Higgs boson, LHC direct SUSY searches, recent evidence for a Standard Model-like BR $\left(B_{s} \rightarrow \mu^{+} \mu^{-}\right)$, and the measurement of $\delta(g-2)_{\mu}$, plus a number of other electroweak and flavor physics constraints. We perform a simulation of two LHC direct SUSY searches at $\sqrt{s}=8 \mathrm{TeV}$ : the CMS inclusive $\alpha_{T}$ search for squarks and gluinos and the CMS electroweak production search with $3 l+E_{T}^{\text {miss }}$ in the final state. We use the latter to identify the regions of the parameter space, consistent at $2 \sigma$ with $\delta(g-2)_{\mu}$, that are not excluded by the direct limits from the electroweak production. We find that they correspond to a neutralino mass in the window $200 \mathrm{GeV} \lesssim m_{\chi} \lesssim 500 \mathrm{GeV}$. We also implement the likelihood for the XENON100 exclusion bound, in which we consider for the first time the impact of a recent determination of the $\Sigma_{\pi N}$ term from CHAOS data, $\Sigma_{\pi N}=43 \pm 12 \mathrm{MeV}$. We show that in light of this measurement, the present statistical impact of the XENON100 bound is greatly reduced, although future sensitivities of the LUX and XENON1T experiments will have decisive impact on the mixed bino/higgsino composition of the neutralino. We point out some tension between the constraints from $\delta(g-2)_{\mu}$ and XENON100. Finally, we present prospects for various indirect searches of dark matter, namely $\gamma$-ray fluxes from dSphs and the Galactic Center at Fermi-LAT, and the positron flux at AMS02. We also show the 5-year sensitivity on the spin-dependent neutralino-proton cross section due to neutrino fluxes from the Sun at IceCube.
\end{abstract}

* On leave of absence from the University of Sheffield, U.K. 


\section{Introduction}

The recent discovery of a particle consistent with the Standard Model (SM) ${ }_{1}^{1}$ Higgs boson at the LHC [1] provides a strong constraint on models of low-scale supersymmetry (SUSY). According to the latest measurements, the mean value of the particle's mass at CMS is $125.7 \pm 0.4 \mathrm{GeV}$ [2] and at ATLAS 125.5 $\pm 0.6 \mathrm{GeV}$ [3]. It was shown in many studies [4, 5, 6, 17, 8, 9, 10, 11] that the predictions of the Minimal Supersymmetric Standard Model (MSSM) for the lightest Higgs boson are consistent with the measured value if large radiative corrections are added to the tree-level mass. If the observed new particle is the lightest $C P$-even Higgs $h$ of the MSSM, $m_{h} \simeq 126 \mathrm{GeV}$ can be achieved, at one loop, either by requiring the stop masses in the multi- TeV range or by requiring maximal stop mixing, $\left|X_{t}\right| / M_{\mathrm{SUSY}} \simeq \sqrt{6}$. Additionally, if one requires that the present observed value of the relic density is due exclusively to neutralino dark matter (DM), the fact that only a limited number of mechanisms can enhance the annihilation cross section up to the value measured by WMAP or PLANCK puts even more severe constraints [12, 13, 14, 15] on the parameter space of models defined at the low-energy SUSY breaking scale, and even more so [16, 17, 18, 19, 20, 21, 22, 23, 24, 25] in SUSY models constrained by universality conditions at the scale of grand unification (GUT), like the Constrained MSSM (CMSSM) [26] and the NonUniversal Higgs Mass model (NUHM) [27]. In particular, it was shown, first in the MSSM [28] and more recently in unified SUSY models [29, 22, 23, 24, 25], that in addition to the previously favored gaugino-like neutralino, the right relic density can be obtained also with a nearly pure higgsino lightest SUSY particle (LSP) with mass around $1 \mathrm{TeV}$.

The above picture is in agreement with the recent positive observation [30] of $\mathrm{BR}\left(B_{s} \rightarrow \mu^{+} \mu^{-}\right)=$ $3.2_{-1.2}^{+1.5} \times 10^{-9}$ at $\mathrm{LHCb}$ : if this value converges in future measurements with higher integrated luminosity to the SM expectation, about $3.6 \times 10^{-9}$ [31, 32], then it was shown in [25] that this measurement will strongly disfavor a substantial part of the CMSSM (specifically, the $A$-funnel (AF) region), unless the value of the pseudoscalar Higgs mass $m_{A}$ is, again, in the multi-TeV regime. The described picture is also in agreement with the nonobservation of SUSY particles at the LHC so far. All of this suggests that if low-energy SUSY exists and takes one of the forms that theorists have hypothesized, it probably comes with masses larger than the energy scale tested by the most recent LHC runs. Recent mass limits calculated with simplified model spectra (SMS), with highly simplified decay chains, which on a case-by-case basis assume only a few SUSY masses in reach of experimental observation whereas the others are decoupled, imply that when the neutralino is very light, the mass of the gluino should be greater than $\sim 1300 \mathrm{GeV}$ [33, 34], the masses of the first- and second-generation squarks should be at least as heavy [34, the masses of thirdgeneration squarks should exceed $600-700 \mathrm{GeV}$ [35, 36, 37, and the mass of the chargino should be greater than $\sim 650 \mathrm{GeV}$ [38]. (We emphasize again that these limits are, however, obtained under assumptions that are, in general, not applicable to realistic SUSY models.)

The only piece of information suggesting the sub-TeV scale of SUSY masses is $\delta(g-2)_{\mu}=$ $(28.7 \pm 8.0) \times 10^{-10}$ [39], which is more than $3 \sigma$ in excess of zero (axiomatically its SM value), and which seems to favor light smuons, muon sneutrinos, charginos, and neutralinos, if the discrepancy is to be explained within SUSY. It has been shown in several studies (see, e.g., [40, 18, 20]) that this cannot be achieved in constrained models, due to the fact that the soft scalar masses for squarks and sleptons are unified at the GUT scale. On the other hand, relaxing some of the unification conditions, so to allow the first-two-generation sleptons to be light, will easily reduce the tension with the $(g-2)_{\mu}$ constraint, and still maintain consistency with the above picture.

Recently, there has been great proliferation of studies investigating the impact of different

\footnotetext{
${ }^{1}$ The abbreviations used throughout the main text of this paper are summarized in Appendix A.
} 
constraints on phenomenological parametrizations of the MSSM. The idea is to treat the $n$ SUSYscale parameters of the MSSM that parametrize the sector of the theory to be studied as free, and constrain the remaining ones by some unifying conditions, or fix them at some decoupled value. Most of the papers on these "pnMSSM" models analyzed the impact of constraints from direct LHC SUSY searches [41, 42, 43, 44, 45, 46, 47], from the Higgs discovery [48, 12, 49, 50, 51, 52, 53, 54], from $b$-physics [55, 56, 57, 58, 54, and from direct and indirect detection of DM [12, 51, 47, 54]. Also, in the past few months there has been a resurgence of interest in scenarios that can reconcile the $(g-2)_{\mu}$ anomaly with the most recent experimental determination from other sectors, like the LHC [59], DM [14], the Higgs [60, and lepton-flavor violation 61, 62] in SUSY, and also in other beyond-the-SM models 63 .

A detailed study of the impact of the experimental constraints described above on the DM sector of a MSSM parametrization with 13 free parameters (p13MSSM) was presented in [15]. The study focused primarily on neutralino DM in the sub-TeV regime, thus only touching the parameter space giving higgsino DM at $1 \mathrm{TeV}$, which has been shown to be an important candidate in studies of GUT-constrained model, as explained at the beginning of this section. We think it would therefore be interesting to also investigate how this sector of the theory agrees with the global set of constraints. Moreover, it has been demonstrated in many studies (see, e.g., 64]) that a proper treatment of the experimental constraints through a likelihood function can lead to significantly different results from scans where such constraints are typically implemented in a more simplified boxlike fashion, with all points accepted when satisfying experimental values within some fixed ranges (e.g., 95\% C.L.), and otherwise rejected.

In this paper we perform a statistical analysis of the MSSM at the SUSY scale with 9 free parameters (p9MSSM), which we identify as a minimal set of parameters to allow good agreement with constraints from the relic density, the Higgs mass and decay rates, $\delta(g-2)_{\mu}, b$-physics constraints, limits from the XENON100 DM direct detection (DD) experiment, limits from LHC direct SUSY searches, and limits from indirect detection (ID) of DM.

To this end we construct approximate but accurate likelihood functions to incorporate in our analysis limits on SUSY from the $\sqrt{s}=8 \mathrm{TeV} \alpha_{T}$ inclusive search for squarks and gluinos with 11.7/fb integrated luminosity [65] and the $\sqrt{s}=8 \mathrm{TeV}, 9.2 / \mathrm{fb}, 3 l+E_{T}^{\text {miss }}$ electroweak (EW) production search at CMS [38]. The latter in particular gives the strongest limits on chargino-neutralino pair production to date, so that our detailed implementation can accurately constrain the sector of the theory consistent with $\delta(g-2)_{\mu}$. Likewise we construct an approximate but accurate likelihood function to incorporate limits on the spin-independent (SI) neutralino-nucleon cross section, $\sigma_{p}^{\mathrm{SI}}$, from XENON100 [66]. We include the theoretical uncertainties due to the determination of the $\Sigma_{\pi N}$ term [67], in light of the recent determination from CHAOS data presented in [68].

We will focus, in particular, on the DM sector of the model, by not only including an accurate implementation of the XENON100 limit, but also by investigating in detail the impact of ID searches: $\gamma$-ray fluxes from the Galactic Center (GC) of the Milky Way and from its dwarf spheroidal satellite galaxies (dSphs) at Fermi-LAT, and positron fluxes at AMS02. We will also try to evaluate the reach of future DM experiments for the parameter space of the model.

The article is organized as follows. In Sec. 2 we briefly revisit the model, highlighting some of its salient features, and detail our methodology, including our statistical approach. In Sec. 3 we describe the relevant experimental results and their likelihood functions, including a detailed discussion of our derivation of the bounds on SUSY from direct searches at the LHC, and of the bound from XENON100. In Sec. 4 we present the results from our scans and discuss their novel features. We summarize our findings in Sec. 5 . 


\section{Model description and numerical methodology}

As mentioned in the Introduction, and shown in many studies (see, e.g., our previous work [20, 69, 25. 70]), the discovery of the Higgs mass has made the multi-TeV scale of $M_{\text {SUSY }} \approx m_{\tilde{t}}$ difficult to avoid in constrained SUSY models (although, given the theoretical uncertainties, in the staucoannihilation region of the CMSSM the mass of the lightest Higgs is consistent with the measured value even if $M_{\mathrm{SUSY}} \simeq 1 \mathrm{TeV}$, thanks to maximal $\left|X_{t}\right| / M_{\mathrm{SUSY}}$ [20, 25]). On the other hand, several groups [49, 55, 71] have shown that in the general MSSM, the parameter space is much less constrained given the large number of free parameters.

In this paper we investigate the impact of the global constraints on the p9MSSM, whose 9 free parameters have been chosen on the basis of their relevance for the constraints involving DM, the Higgs sector, and other relevant quantities. In this section, we will describe our p9MSSM parameter choice.

We make some reasonable and simplifying assumptions, as is usually done in the MSSM. First, we assume universality for the bino and wino masses, $M_{1}=0.5 M_{2}$, resulting in the absence of wino-like neutralino as the LSP. It is known that a wino-like neutralino can hardly satisfy the relic density constraint, unless taken to be very heavy $\left(m_{\chi} \gtrsim 1.6 \mathrm{TeV}[28,72\right.$ ] but see [47] for very recent numerical work on the issue), and it has been shown to be in potential conflict with ID experiments due to its large annihilation cross section [73. On the other hand, in order to mitigate possible impacts on the DM sector from LHC multi-jet limits, we treat the gluino mass $M_{3} \approx m_{\tilde{g}}$ as a free parameter. We scan it in the range $0.7 \mathrm{TeV}<M_{3}<8 \mathrm{TeV}$ since lower values are now disfavored by most LHC direct SUSY searches.

The squarks of the first two generations are strongly constrained by direct searches at the LHC and are basically irrelevant for the constraints that we will employ. We therefore fix them at a decoupled scale, $m_{\tilde{Q}_{1,2}}=m_{\tilde{u}_{R}^{(1,2)}}=m_{\tilde{d}_{R}^{(1,2)}}=2.5 \mathrm{TeV}$. Instead, we allow wide ranges for the thirdgeneration squark masses, $m_{\tilde{Q}_{3}}=m_{\tilde{t}_{R}}=m_{\tilde{b}_{R}}$, because they are not constrained by the LHC as strongly as the first and second generations, and they affect the Higgs sector.

In order to save computer time and make sure we do not generate many points with charged LSP, which would then be rejected, we unify the first- and second-generation sleptons and set them to $m_{\tilde{L}_{1,2}}=m_{\tilde{e}_{R}}=m_{\tilde{\mu}_{R}}=M_{1}+50 \mathrm{GeV}$. It will be shown that, when the relic density constraint is taken into account, this choice does not compromise good coverage of the sector related to $(g-2)_{\mu}$.

On the other hand, we scan over $m_{\tilde{L}_{3}}=m_{\tilde{\tau}_{R}}$ to investigate scenarios where the relic density is obtained via stau-coannihilation.

We unify all the lepton trilinear couplings to $A_{e}=A_{\mu}=A_{\tau}$. All up-type squark trilinear couplings are unified to $A_{t}$ but down-type squark trilinear couplings are fixed to $A_{b}=-0.5 \mathrm{TeV}$, since our likelihood function will not be sensitive to down-type squark trilinear couplings. We allow broad ranges for $A_{t}$ and $A_{\tau}$ to investigate the Higgs sector and mechanisms for stau-coannihilation, respectively.

We investigate the Higgs sector by scanning over $m_{A}$, the Higgs/higgsino mass parameter $\mu$, and the ratio of the Higgs doublets' vacuum expectation values (vev), $\tan \beta$.

In summary, our multi-dimensional scan is parametrized by 9 free SUSY parameters:

$$
M_{2}, M_{3}, m_{\tilde{Q}_{3}}, m_{\tilde{L}_{3}}, A_{t}, A_{\tau}, m_{A}, \mu, \tan \beta .
$$

In addition, we scan over the bottom quark mass, $m_{b}\left(m_{b}\right)^{\overline{M S}}$, and top quark pole mass, $M_{t}$, to include SM uncertainties. In contrast to our previous papers, we do not vary the strong interaction coupling, $\alpha_{s}$, or the electromagnetic coupling $\alpha_{\mathrm{em}}$ because they are well constrained. Moreover, $\alpha_{\mathrm{em}}$ is used in CMSSM and NUHM scans to set the GUT scale, which is not an issue in here, 


\begin{tabular}{|c|c|}
\hline \hline Parameter & Range \\
\hline \hline gluino mass & $0.7<M_{3}<8$ \\
wino mass & $0.01<M_{2}<4$ \\
bino mass & $M_{1}=0.5 M_{2}$ \\
stop trilinear coupl. & $-7<A_{t}<7$ \\
$\tau$ trilinear coupl. & $-7<A_{\tau}<7$ \\
sbottom trilinear coupl. & $A_{b}=-0.5$ \\
pseudoscalar mass & $0.2<m_{A}<4$ \\
$\mu$ parameter & $0.01<\mu<4$ \\
3rd gen. soft squark mass & $0.3<m_{\tilde{Q}_{3}}<4$ \\
3rd gen. soft slepton mass & $0.1<m_{\tilde{L}_{3}}<2$ \\
1st/2nd gen. soft slepton mass & $m_{\tilde{L}_{1,2}}=M_{1}+50 \mathrm{GeV}$ \\
1st/2nd gen. soft squark mass & $m_{\tilde{Q}_{1,2}}=2.5$ \\
ratio of Higgs doublet VEVs & $3<$ tan $\beta<62$ \\
\hline Nuisance parameter & Central value, error \\
\hline \hline Bottom mass $m_{b}\left(m_{b}\right)^{M S}(\mathrm{GeV})$ & $(4.18,0.03)$ \\
Top pole mass $M_{t}(\mathrm{GeV})$ & $(173.5,1.0)$ \\
\hline \hline
\end{tabular}

Table 1: Prior ranges for our p9MSSM input parameters, over which we perform our scan. All masses and trilinear couplings are in $\mathrm{TeV}$, unless indicated otherwise.

since soft SUSY-breaking parameters are defined at the scale $M_{\mathrm{SUSY}}=\sqrt{m_{\tilde{t}_{1}} m_{\tilde{t}_{2}}}$, which is found iteratively by our RGE code (softsusy-3.3.5 [74]).

The set of input parameters we consider in this paper and their scanned ranges are shown in Table 1 .

We perform our scans by using the package BayesFITS, which interfaces different publicly available programs. For sampling, it uses MultiNest [75] with 20000 live points, evidence tolerance factor equal to $10^{-4}$, and sampling efficiency equal to 0.8 . We compute mass spectra and $m_{W}$ with softsusy-3.3.5 [74] and pass the spectra via SUSY LesHouches Accord to superiso v3.3 [76] to calculate $\mathrm{BR}\left(\bar{B} \rightarrow X_{s} \gamma\right), \mathrm{BR}\left(B_{s} \rightarrow \mu^{+} \mu^{-}\right)$, BR $\left(B_{u} \rightarrow \tau \nu\right)$, and $\delta(g-2)_{\mu}^{\mathrm{SUSY}}$. DM observables, such as the relic density and DD and ID observables, are calculated with MicrOMEGAs 2.4.5 [77] and DarkSUSY [78]. Higgs cross sections, $\Delta M_{B_{s}}$, and $\sin \theta_{\text {eff }}$ are computed with FeynHiggs 2.9.4 [79, 80, 81, 82. In addition, we also check the exclusion bounds obtained from the Higgs searches at LEP and the Tevatron with HiggsBounds 3.8.0 [83].

Note that MultiNest is optimized for Bayesian sampling. The scans are driven by the likelihood function, and the input parameters are subject to prior distributions. We combine six separate scans: three with log priors in the mass parameters (with the exclusion of $\mu$ ) and three with flat priors, so to obtain good coverage of the parameter space. We take flat priors for the trilinear couplings, for $\mu$, and for $\tan \beta$ in all six scans. Moreover, the nuisance parameters are always scanned over with Gaussian prior distributions.

We employ in this work the profile-likelihood approach, which we briefly summarize in the next section, to draw inferences on the multi-dimensional parameter space of the p9MSSM. The advantage of the profile-likelihood method with respect to our previous papers [84, 20, 69], where we were calculating Bayesian inferences based on the posterior probability density function, is that we can merge together many chains with different priors to explore the whole parameter space in 


\begin{tabular}{|c|c|c|c|c|}
\hline Measurement & Mean or range & Error: exp., th. & Distribution & Ref. \\
\hline $\mathrm{CMS} \alpha_{T} 11.7 / \mathrm{fb}, \sqrt{s}=8 \mathrm{TeV}$ & See text. & See text. & Poisson & 65 \\
\hline$m_{h}($ by CMS $)$ & $125.8 \mathrm{GeV}$ & $0.6 \mathrm{GeV}, 3 \mathrm{GeV}$ & Gaussian & 86 \\
\hline$\Omega_{\chi} h^{2}$ & 0.1199 & $0.0027,10 \%$ & Gaussian & 87 \\
\hline$\widehat{\mathrm{BR}}\left(\bar{B} \rightarrow X_{s} \gamma\right) \times 10^{4}$ & 3.43 & $0.22,0.21$ & Gaussian & 88 \\
\hline $\mathrm{BR}\left(B_{u} \rightarrow \tau \nu\right) \times 10^{4}$ & 1.66 & $0.33,0.38$ & Gaussian & 89 \\
\hline$\Delta M_{B_{s}}$ & $17.719 \mathrm{ps}^{-1}$ & $0.043 \mathrm{ps}^{-1}, 2.400 \mathrm{ps}^{-1}$ & Gaussian & 90 \\
\hline $\sin ^{2} \theta_{\text {eff }}$ & 0.23146 & $0.00012,0.00015$ & Gaussian & 90 \\
\hline$M_{W}$ & $80.399 \mathrm{GeV}$ & $0.023 \mathrm{GeV}, 0.015 \mathrm{GeV}$ & Gaussian & 90 \\
\hline $\mathrm{BR}\left(B_{s} \rightarrow \mu^{+} \mu^{-}\right) \times 10^{9}$ & 3.2 & $+1.5,-1.2,10 \%$ & Gaussian & 30 \\
\hline$m_{b}\left(m_{b}\right)^{\overline{M S}}$ & $4.18 \mathrm{GeV}$ & $0.03 \mathrm{GeV}, 0$ & Gaussian & 90 \\
\hline$M_{t}$ & $173.5 \mathrm{GeV}$ & $1.0 \mathrm{GeV}, 0$ & Gaussian & 90 \\
\hline$\delta(g-2)_{\mu}^{\mathrm{SUSY}} \times 10^{10}$ & 28.7 & $8.0,1.0$ & Gaussian & 39,91 \\
\hline XENON100 (2012) & See text. & See text. & Poisson & 66 \\
\hline CMS $3 l+E_{T}^{\text {miss }} 9.2 / \mathrm{fb}, \sqrt{s}=8 \mathrm{TeV}$ & See text. & See text. & Poisson & 38 \\
\hline
\end{tabular}

Table 2: The experimental constraints that we include in our likelihood functions to constrain our p9MSSM model. We denote the first block of constraints as basic.

good detail, without worrying about appropriate prior weights.

\section{Statistical treatment of experimental constraints}

In this section we briefly describe the profile-likelihood method and the experimental constraints used in this analysis. We will later show our results as $68 \%(1 \sigma)$ and $95 \%(2 \sigma)$, or $90 \%(1.65 \sigma)$ confidence intervals in the p9MSSM parameter space.

For a theory described by a set of $n$ parameters $m$, one can compare experimental observables $\xi(m)$ with data $d$ through the likelihood function $\mathcal{L}(m) \equiv p(d \mid \xi(m))$, which at any point $m$ in parameter space gives the probability of the data $d$ given $m$.

One can draw inference on a subset of $r \leq n$ specific model parameters or observables, or a combination of both (collectively denoted by $\psi_{i}$ ), by "profiling" the likelihood along the other directions in the parameter space [85, 64],

$$
\mathcal{L}\left(\psi_{i=1, . ., r}\right)=\max _{m \in \mathbb{R}^{n-r}} \mathcal{L}(m)
$$

Confidence intervals are calculated from tabulated values of $\delta \chi^{2} \equiv-2 \ln \left(\mathcal{L} / \mathcal{L}_{\max }\right)$. For example, in $r=2$ dimensions, $68.3 \%$ confidence regions are given by $\delta \chi^{2}=2.30$ and $95.0 \%$ confidence regions by $\delta \chi^{2}=5.99$.

The constraints that we include in the likelihood function are listed in Table 2, For the purpose of presentation, we denote the constraints in the first block of the table as basic, as we shall explain in Sec. 4. As a rule, following the procedure developed earlier [92], we implement positive measurements through a Gaussian likelihood, in which the experimental and theoretical uncertainties are added in quadrature. For the Higgs mass, we use the most recent CMS determination of its central value and experimental uncertainty, as it is in good agreement with the determination obtained by ATLAS at the end of the $\sqrt{s}=8 \mathrm{TeV}$ run. The theoretical uncertainty is estimated to be $3 \mathrm{GeV}$ [20, 6]. For the relic density we use the recent determination by PLANCK [87].

Additionally, we impose 95\% C.L. lower limits from direct searches at LEP [90], smeared with $5 \%$ theoretical errors, for the following particles: 


$$
\begin{aligned}
m_{\chi} & >46 \mathrm{GeV}, \\
m_{\tilde{e}} & >107 \mathrm{GeV}, \\
m_{\chi_{1}^{ \pm}} & >94 \mathrm{GeV} \text { if } m_{\chi_{1}^{ \pm}}-m_{\chi}>3 \mathrm{GeV} \text { and } \tan \beta<40, \\
m_{\tilde{\mu}} & >94 \mathrm{GeV} \text { if } m_{\tilde{\mu}}-m_{\chi}>10 \mathrm{GeV} \text { and } \tan \beta<40, \\
m_{\tilde{\tau}} & >81.9 \mathrm{GeV} \text { if } m_{\tilde{\tau}_{1}}-m_{\chi}>15 \mathrm{GeV}, \\
m_{\tilde{b}_{1}} & >89 \mathrm{GeV} \text { if } m_{\tilde{b}_{1}}-m_{\chi}>8 \mathrm{GeV} \\
m_{\tilde{t}_{1}} & >95.7 \mathrm{GeV} \text { if } m_{\tilde{t}_{1}}-m_{\chi}>10 \mathrm{GeV} .
\end{aligned}
$$

The MSSM predictions for $\mathrm{BR}\left(B_{s} \rightarrow \mu^{+} \mu^{-}\right)$must be matched with what was measured by the LHCb experiment, i.e., the time-averaged, flavor-averaged branching ratio, whose measured value is given in Table 2. This averaging incorporates oscillations between the $B_{s}$ and $\bar{B}_{s}$ flavor eigenstates between the primary vertex and the secondary decay vertex, which occur in the experiment. This effect is nontrivial, because the flavor eigenstates have different decay widths. Therefore, to connect with the experiment, one should multiply the theoretical prediction by a factor that takes into account this effect [93, 94], so that

$$
\mathrm{BR}\left(B_{s} \rightarrow \mu^{+} \mu^{-}\right)_{\text {time ave }}=\operatorname{corr} \cdot \mathrm{BR}\left(B_{s} \rightarrow \mu^{+} \mu^{-}\right)_{\text {theory }},
$$

where the "correction" factor is given, for example, in Ref. [56].

SuperIso gives $\mathrm{BR}\left(B_{s} \rightarrow \mu^{+} \mu^{-}\right)$time ave $=3.87 \times 10^{-9}$, which is higher than the most recent time-averaged calculation, $\mathrm{BR}\left(B_{s} \rightarrow \mu^{+} \mu^{-}\right)_{\text {time ave }}^{\mathrm{SM}}=(3.56 \pm 0.18) \times 10^{-9}[32]$. The difference in the two values is due to differences in the chosen value of $f_{B_{s}}$ and other input parameters such as the $B_{s}$ lifetime, the top mass, and the CKM elements. Other sources of uncertainty arise from the chosen renormalization scheme for $\sin \theta_{W}$ and $M_{t}$ [95. Since the uncertainties enter multiplicatively in Eq. (3) as a correction factor and we assume a theoretical uncertainty of $10 \%$ in our scans, we use the non time-averaged output of SuperIso, which gives in the SM limit $\mathrm{BR}\left(B_{s} \rightarrow \mu^{+} \mu^{-}\right)^{\mathrm{SM}}=3.53 \times 10^{-9}$, consistent with the determination of [32] within $1 \sigma$. In addition, since the experimental uncertainty in $\operatorname{BR}\left(B_{s} \rightarrow \mu^{+} \mu^{-}\right)$is asymmetric, we parametrize the likelihood function as a combination of two half-Gaussians, one for positive and negative error each.

In Table 2, $m_{h}$ refers to the mass of the lightest $C P$-even Higgs boson of the model. In principle, it is possible for the heavier $C P$-even Higgs, $H$, to be SM-like with mass $\sim 126 \mathrm{GeV}$, in the so-called nondecoupling regime of the model. However, such a possibility is confined to a very fine-tuned region of the parameter space, which is essentially ruled out by the direct search limits on the lightest Higgs $h$ and the pseudo-scalar Higgs $A$ and other experimental constraints from flavor physics [10, 96, 97, 98]. Hence we do not explore such a possibility here.

The construction of the likelihood for limits from direct SUSY searches and the likelihood for XENON100 deserve a more detailed explanation, which we give in the following subsections.

\subsection{Likelihood for LHC direct SUSY searches}

In order to implement the impact of direct SUSY search limits on the p9MSSM parameter space, we extend the procedure developed previously in [20, 25] for the derivation of approximate but accurate likelihood maps. For the first time, in this study we apply the SUSY likelihood functions on-the-fly, point by point in our scan. Note that in our previous studies of constrained models [20, 69, 25] we relied on likelihood maps prepared separately for subsets of input parameters. 


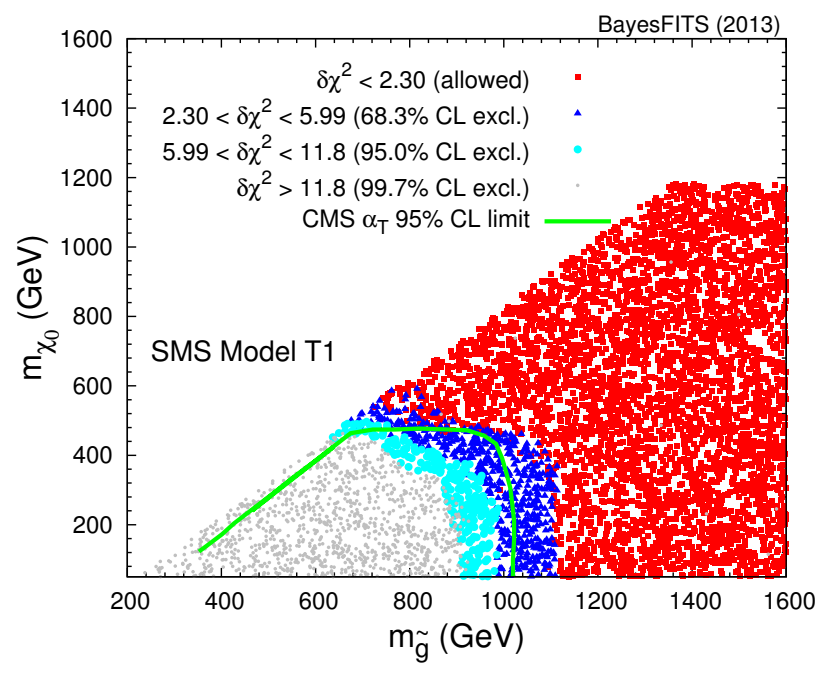

(a)

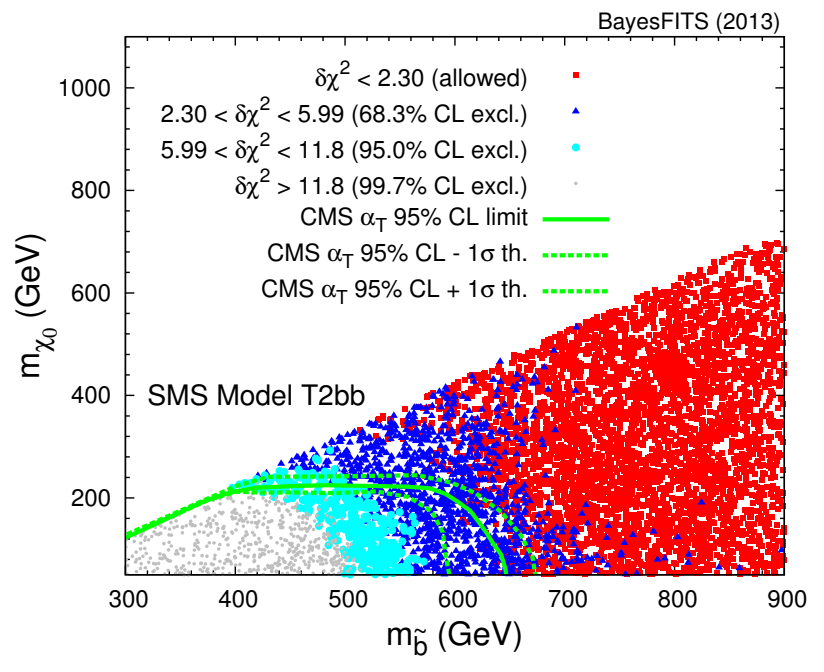

(b)

Figure 1: Our $\alpha_{T}$ likelihood as a function of sparticle masses in (a) SMS Model T1 and (b) SMS Model T2bb. Points that are excluded at $3 \sigma$ are shown as gray dots, at $2 \sigma$ as cyan circles, and at $1 \sigma$ as blue triangles. The $95 \%$ C.L. limit from CMS (solid green line) is also shown to facilitate a comparison with our result (boundary of cyan circles and blue triangles).

In this paper we apply the results from two different searches: CMS inclusive search for SUSY final states with large missing transverse energy and $b$-quark jets in $\sqrt{s}=8 \mathrm{TeV} p p$ collisions with the variable $\alpha_{T}$ [65]; and direct EW production of charginos and neutralinos in $p p$ collisions at $\sqrt{s}=8 \mathrm{TeV}$ [38. Each search is implemented through a step-by-step procedure that includes generation of a SUSY signal at the scattering level with PYTHIA6.4 [99] and a simulation of the CMS detector response with PGS4 [100] to calculate the efficiency once the kinematic cuts are applied. We modified the CMS detector card as recommended by the CMS Collaboration, and we tuned the algorithm used by PGS4 to reproduce the $b$-tagging efficiency reported by CMS. The obtained signal yields are finally statistically compared to the publicly available observed and background yields of the searches, provided by the CMS Collaboration, as described in [25], which updated the procedure described in detail in [84, 20].

CMS $\alpha_{T} \mathbf{1 1 . 7} / \mathbf{f b}, \sqrt{s}=8 \mathrm{TeV}$

The search employs a set of 8 different boxes, with hard jets and missing energy in the final states, and different combinations of $b$-tagged jets. The boxes with zero $b$-tagged jets in the final states are used to impose limits on the squarks of the first two generations produced either directly or in gluino decays. Increasing numbers of $b$-tagged jets in the final state implies direct production of third generation squarks, or gluinos decaying to the latter. The boxes, together with the number of the observed and background events provided by the CMS Collaboration, are given in [101].

We validate our procedure against the official experimental limits in the framework of SMS. In Fig. 1](a) we present the bounds obtained from our likelihood in the gluino-LSP plane for model T1 of pair-produced gluinos decaying into a neutralino and quarks of the first two generations [65]. In Fig. 1 $1(\mathrm{~b})$ we show the bounds in the sbottom-LSP plane for model T2bb for pair-produced bottom squarks. Gray dots represent the points excluded by our likelihood function at the $99.7 \%$ C.L., cyan 


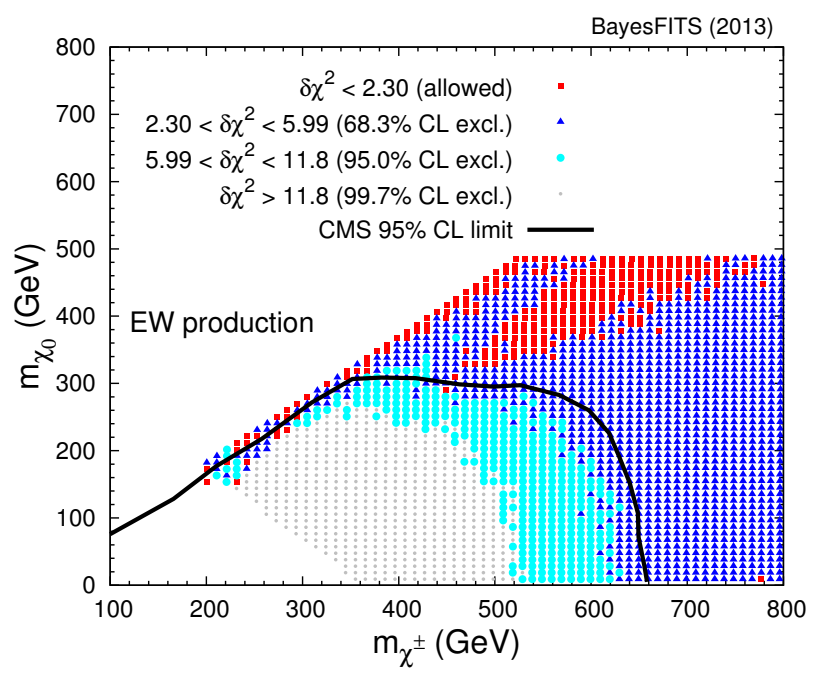

Figure 2: Our EW-production likelihood as a function of neutralino and chargino masses. Points that are excluded at $3 \sigma$ are shown as gray dots, at $2 \sigma$ as cyan circles, and at $1 \sigma$ as blue triangles. The $95 \%$ C.L. limit from CMS (solid black line) is also shown to facilitate a comparison with our result (boundary of cyan circles and blue triangles).

circles those excluded at the $95.0 \%$ C.L., and blue triangles those excluded at the $68.3 \%$ C.L. The remaining points (red squares) are considered as allowed. The solid green line shows the official 95\% C.L. CMS exclusion limit.

As one can see, we reproduce the experimental exclusion bound in model T1 very well, with a discrepancy in the gluino mass limit that stays below $50 \mathrm{GeV}$ for most of the parameter space, although it can be twice as large for $m_{\chi} \simeq 400 \mathrm{GeV}$. The agreement is slightly worse for model T2bb, where the discrepancy is about $70 \mathrm{GeV}$. The reason is that we use in our analysis the cross section at the leading order, as provided by PYTHIA6.4, which differs from the cross section implemented in the experimental analysis by a factor of around 1.8. Also note that the theoretical sbottom production cross section is subject to a large theoretical uncertainty (shown in Fig. 1](b) as dashed green lines) that could affect the determination of the experimental 95\% C.L. line by around $60 \mathrm{GeV}$. It is important to keep in mind that such uncertainties will not have a significant impact on the determination of the profile-likelihood confidence regions and the best-fit points, as the other constraints favor the multi- TeV region for squarks and gluinos.

We apply the described procedure to calculate the $\alpha_{T}$-given $\delta \chi^{2}$ of all the points of the p9MSSM sensitive to the search (about $1 \%$ of the total set) ${ }^{2}$ One just needs to bear in mind that the $\alpha_{T}$ constraints presented in this paper are conservative.

Although the $\sqrt{s}=8 \mathrm{TeV} \alpha_{T}$ search imposes strong limits on the masses of third-generation squarks in the p9MSSM, very recently the ATLAS and CMS collaborations updated their results for direct top squark pair production [35, 37] with the analyses based on $20.7 / \mathrm{fb}$ and $19.5 / \mathrm{fb}$ of data, respectively. We will test at the end the consistency of our best-fit point with the most updated ATLAS and CMS third-generation searches.

\footnotetext{
${ }^{2}$ With respect to the SMS used for validation, the PYTHIA card is changed for the full scan to include inclusive production.
} 
CMS $3 l+E_{T}^{\text {miss }} \mathbf{9 . 2} / \mathbf{f b}, \sqrt{s}=8 \mathrm{TeV}$ (EW production)

We follow the same procedure to construct the likelihood function for chargino-neutralino pair production (EW production). We use the CMS search [38], which currently gives the strongest direct limits.

We consider the channels with three leptons in the final state: an opposite-sign-same-flavor lepton pair, ee or $\mu \mu$, and a third lepton being either an electron or a muon. We have checked on a few test scans that these channels yield the highest sensitivity. The observed and background events are given in Table 1 of [38]. We validate our procedure against the official CMS 95\% C.L. exclusion bound for a SMS with $m_{\tilde{l}}=0.5 m_{\chi}+0.5 m_{\chi_{1}^{ \pm}}$38]. The result is shown in Fig. 2, where the color code is the same as in Fig. 1. As one can see the discrepancy in the chargino mass bound is less than $50 \mathrm{GeV}$ for the neutralino mass range.

Given our parameter choice and ranges, the likelihood for EW production of neutralinos and charginos can rule out a large fraction of the total number of scanned points. Even by preliminarily considering only the points that can satisfy all other constraints, the number of points in our scans that can be potentially affected by the EW-production likelihood is about 400,000 (out of 1.8 millions in total). For this reason, including this search in the global likelihood function has proven to be a numerically unmanageable task. Thus, the contribution to the $\chi^{2}$ of this search is calculated on a randomly chosen sample of approximately 40,000 points from our chains. This will be enough to draw general conclusions. We will use this information to check the consistency of this search with the other constraints (and particularly $\delta(g-2)_{\mu}$ ), and also we will test the best-fit points from the global likelihood against EW production to make sure that they are not excluded.

\subsection{XENON100 likelihood}

A proper treatment of the XENON100 90\% C.L. bound in the $\left(m_{\chi}, \sigma_{p}^{\mathrm{SI}}\right)$ plane [102, 103, 104] is not straightforward because, as it has been long known, the limits from DD of DM experiments on SUSY parameter space are marred by large nuclear physics uncertainties [105, 106]. The astrophysics uncertainties resulting from the DM local density and velocity distribution, on the other hand, affect the elastic scattering cross section by only around $50 \%$ in the mass range considered in this paper [107].

Nuclear physics uncertainties enter the picture through the calculation of the cross section of DM-quark elastic scattering. To connect this prediction with experiment one has to estimate a nucleon mass matrix, $\langle N|\bar{q} q| N\rangle$, to transform the cross section from the quark level to the nucleon level. The nucleon mass matrix calculation is subject to uncertainties on the quark masses $m_{d, c, b, t}$, on the ratios $m_{u} / m_{d}$ and $m_{s} / m_{d}$, and on the hadronic quantities related to the change in the nucleon mass due to nonzero quark masses, $\sigma_{0}$ and $\Sigma_{\pi N}$ :

$$
\begin{aligned}
\sigma_{0} & =\frac{m_{u}+m_{d}}{2}\langle N|\bar{u} u+\bar{d} d-2 \bar{s} s| N\rangle, \\
\Sigma_{\pi N} & =\frac{m_{u}+m_{d}}{2}\langle N|\bar{u} u+\bar{d} d| N\rangle .
\end{aligned}
$$

$\Sigma_{\pi N}$ is generally derived by extrapolating information from experimental input, generally $\pi-N$ elastic scattering cross sections. It has been long known that these hadronic uncertainties can be much larger than astrophysical uncertainties (see [108] and references therein).

In a recent paper [68], the differential elastic $\pi-N$ scattering cross sections [109] measured with the CHAOS detector at TRIUMF [110] were employed to derive a new determination of the $\Sigma_{\pi N}$ term, $\Sigma_{\pi N}=43 \pm 12 \mathrm{MeV}$, where the error bar is mostly due to the experimental uncertainties. 


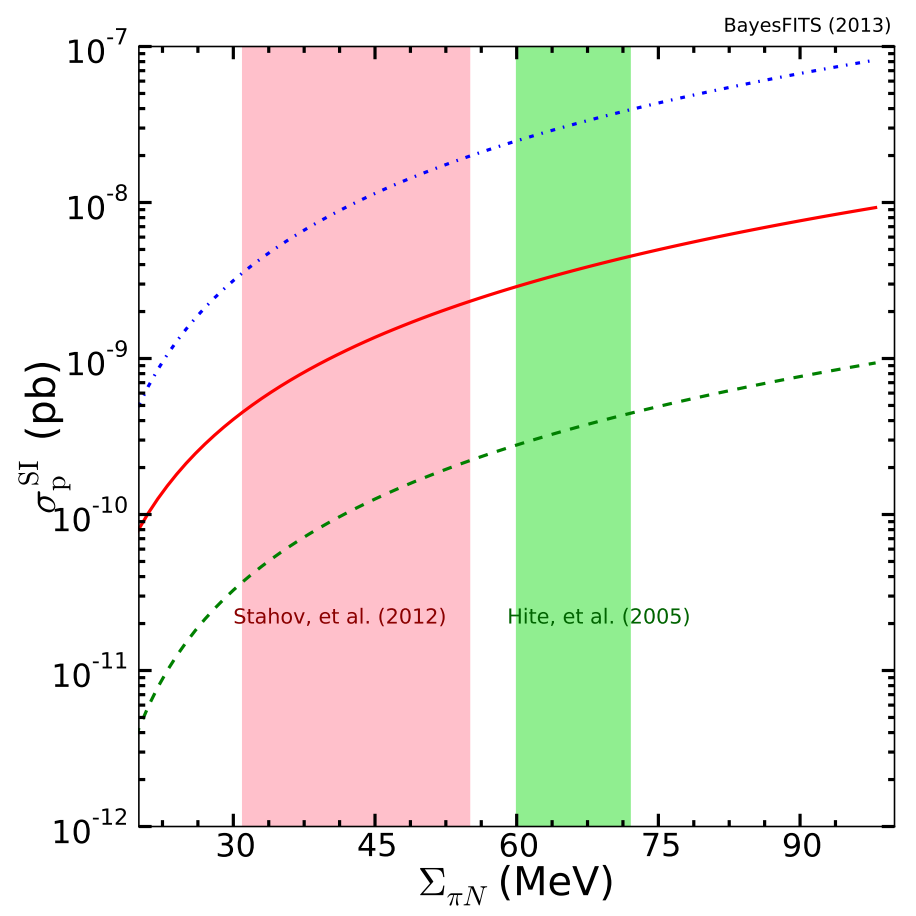

Figure 3: The spin-independent neutralino-proton scattering cross section versus the pion-nucleon $\Sigma$ term for three p9MSSM points (shown in Table 3), characterised by their neutralino composition: the dash-dotted blue line shows a point corresponding to mixed neutralino, the solid red line to a higgsino-like neutralino, and the dashed green line to a gaugino-like neutralino. $1 \sigma$ confidence intervals for the pion-nucleon $\Sigma$ term from [68] (light red, left) and [113] (light green, right) are shown by vertical shaded blocks.

This value is substantially lower than the values previously calculated using phase-shift analyses from the GWU/SAID database [111], or using chiral perturbation theory [112], and it can have substantial implications, as we shall see, when deriving limits on SUSY from DD experiments.

To illustrate this point, we show in Fig. 3 the dependence of the SI cross section on the $\Sigma_{\pi N}$ term for three different neutralino masses and gaugino/higgsino fractions. One can see that $\sigma_{p}^{\mathrm{SI}}$ can vary by more than one order of magnitude over the plotted range of $\Sigma_{\pi N}$, and by a factor of five over the $1 \sigma$ range of [68] (light red band on the left). Thus, in this study we include the most recent $\Sigma_{\pi N}$ determination of [68] (with its uncertainties) in the likelihood function for XENON100.

The likelihood function for XENON100 is given by the product of an experimental and a theoretical part. We build the experimental, model-independent part following the procedure described in detail in Sec. IIIB of Ref. [114. We assume that number of observed events follows a Poisson distribution about the number of "signal+background" events. The systematic uncertainties are parametrized by marginalizing the background prediction with a Gaussian distribution of mean $b=1$ and standard deviation $\delta b=0.2$, as given by the XENON Collaboration [66]. An "exclusion signal," $s_{90}^{*}$, is thus calculated,

$$
0.1=\frac{\int_{s_{90}^{*}}^{\infty} \mathcal{P}\left(s^{\prime}+b \mid o\right) d s^{\prime}}{\int_{0}^{\infty} \mathcal{P}\left(s^{\prime}+b \mid o\right) d s^{\prime}},
$$


where the probability distribution is given by

$$
\mathcal{P}(s+b \mid o)=\int_{0}^{\infty} \frac{e^{-\left(s+b^{\prime}\right)}\left(s+b^{\prime}\right)^{o}}{o !} \exp \left[-\frac{\left(b^{\prime}-b\right)^{2}}{2 \delta b^{2}}\right] d b^{\prime},
$$

and $o=2$ is the number of observed events $[66$.

For each pair $\left(m_{\chi}, \sigma_{p, 90}^{\mathrm{SI}}\right)$ lying on the $90 \%$ C.L. exclusion line, a signal $s_{\mathrm{mo}}$ is then calculated with micrOMEGAs, in the nuclear recoil energy range of $6.6-30.5 \mathrm{keV}_{\mathrm{nr}}$. We shall use the default setting of DM velocity distribution (the truncated Maxwell distribution). One can thus derive experimental "efficiencies,"

$$
\varepsilon\left(m_{\chi}, \sigma_{p, 90}^{\mathrm{SI}}\right)=\frac{s_{90}^{*}}{s_{\mathrm{mo}}\left(m_{\chi}, \sigma_{p, 90}^{\mathrm{SI}}\right)},
$$

which account for the experimental acceptances.

We finally account for nuclear physics uncertainties by profiling over $\Sigma_{\pi N}$ with Gaussian distribution,

$$
\mathcal{L}\left[m_{\chi}, \sigma_{p}^{\mathrm{SI}}\left(\Sigma_{\pi N}\right)\right]=\max _{\Sigma_{\pi N}^{\prime}} \mathcal{P}\left[\varepsilon s_{\mathrm{mo}}\left(m_{\chi}, \sigma_{p}^{\mathrm{SI}}\left(\Sigma_{\pi N}^{\prime}\right)\right)+b \mid o\right] \cdot \exp \left[-\frac{\left(\Sigma_{\pi N}^{\prime}-\Sigma_{\pi N}\right)^{2}}{2 \sigma_{\Sigma_{\pi N}}^{2}}\right],
$$

where the probability $\mathcal{P}$ is given in Eq. (6). Here, we only vary $\Sigma_{\pi N}$ as the largest source of uncertainty but fix other mass matrix parameters, such as $m_{u} / m_{d}=0.553, m_{s} / m_{d}=18.9$ and $\sigma_{0}=35.5 \mathrm{MeV}$.

In Fig. Al(a), we show the impact of incorporating the $\Sigma_{\pi N}$ uncertainties into the likelihood. All the points in the plot satisfy the constraints from LEP, $\alpha_{T}$ limits, Higgs mass, PLANCK, and flavor physics. The points excluded by the likelihood function of Eq. (8) at the $90 \%$ C.L. $\left(\delta \chi^{2}=4.61\right)$ are shown as gray crosses and the rest of the points as yellow circles. Note that, since we profile on the theoretical uncertainty, the position of the points in the plane can float according to the value of $\Sigma_{\pi N}$ producing the largest likelihood, see Eq. (8). One can see that, given the new determination and uncertainties on $\Sigma_{\pi N}$, the impact of the XENON100 constraint on the parameter space of the p9MSSM is almost negligible.

In contrast, we repeated the same procedure by considering a recent determination $\Sigma_{\pi N}=$ $66 \pm 6 \mathrm{MeV}$ [113, 115] obtained from the most recent GWU pion-nucleon phase-shift analysis [116]. One can see in Fig. 4|(b) that in this case a substantial fraction of points is excluded by XENON100.

In our results we will show the effect of applying in turn one or the other determination, and discuss the ensuing implications for the p9MSSM.

\section{Results}

We collected a total of about $1.8 \times 10^{6}$ points through several scans of the p9MSSM parameter space, as defined in the previous sections.

We identify three different sets of constraints, which are shown separately in Table 2. The upper box encapsulates what we define as the basic set of constraints, which are taken into account in all of the plots presented below. On the other hand, the $(g-2)_{\mu}$ and XENON100 constraints are included in the global likelihood when discussing the impact of these specific constraints, and we will indicate explicitly in the text and figures when this is the case. Finally, the EW-production constraint is treated separately, as this contribution is added to the likelihood only for a randomly chosen thinned selection of points, as explained in Sec. 3.1 . 


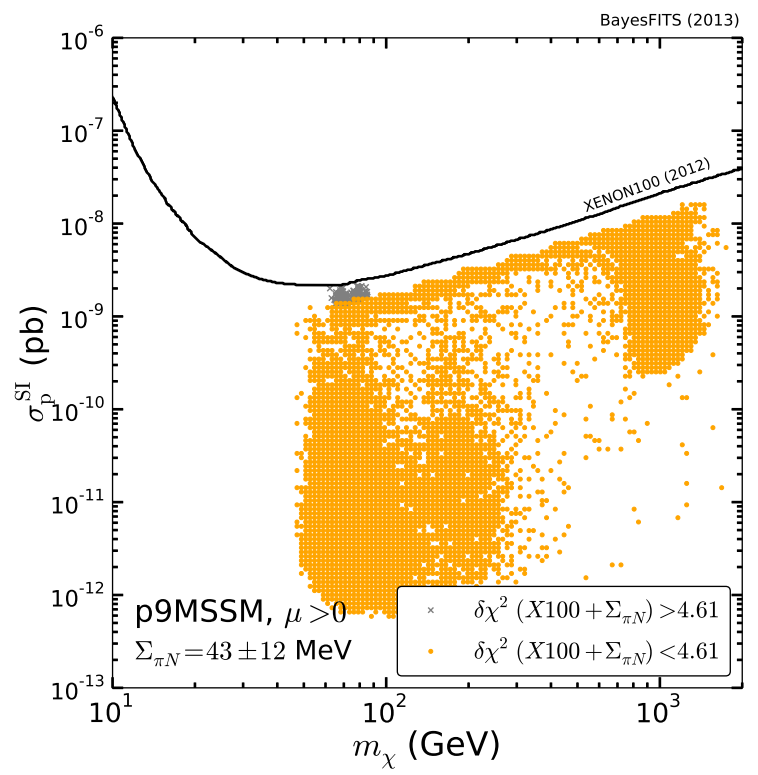

(a)

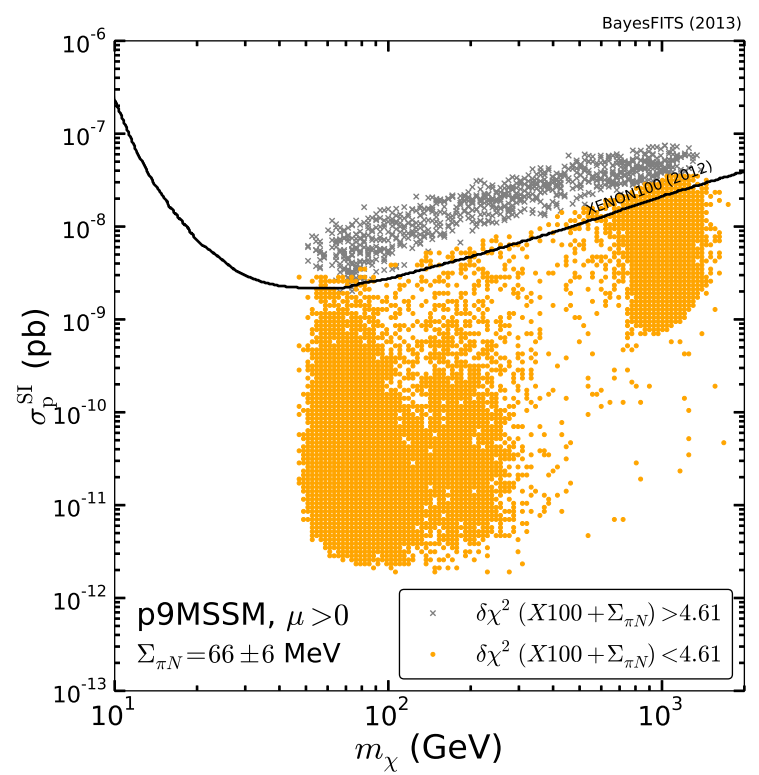

(b)

Figure 4: p9MSSM points scattered in the $\left(m_{\chi}, \sigma_{p}^{\mathrm{SI}}\right)$ plane assuming (a) $\Sigma_{\pi N}=43 \pm 12 \mathrm{MeV}$ and (b) $\Sigma_{\pi N}=66 \pm 6 \mathrm{MeV}$. The XENON100 90\% C.L. exclusion contour is shown in solid black. Points excluded through the likelihood of Eq. (8) at the 90\% C.L. $\left(\delta \chi^{2}>4.61\right)$ are shown as gray crosses. All the points in the plots satisfy the constraints from LEP, $\alpha_{T}$, Higgs mass, PLANCK, and flavor physics.

In Fig. 5| (a), we show the distribution of our points in the $\left(M_{1}, \mu\right)$ plane. The gray dots are excluded at the $95 \%$ C.L. based on the profile-likelihood method, after applying the basic set of constraints $\left(\delta \chi_{\text {basic }}^{2}>5.99\right)$. In the remainder of this section, we will not show the gray dots again, i.e., we will present our results as $95 \%$ confidence regions in two-dimensional (2D) projections based on the profile-likelihood method. However, before we move on, we want to point out a couple of features of the gray dots. The first is that their distribution appears in the plot to lie on a grid. The reason is that, although all of our $>10^{6}$ points are generated randomly, we binned the data excluded at the $95 \%$ C.L. in a $100 \times 100$-step grid to reduce the size of the picture. The second is that some of the points are missing from the upper right corner of Fig. 馬(a), The reason has to do with our choice of parameter ranges (Table 1): those points are characterized by a neutralino mass around $2000 \mathrm{GeV}$, and the upper limit on the stau mass is also $\sim 2000 \mathrm{GeV}$. Thus, in that region MultiNest is likely to generate points with stau LSP, which are automatically rejected.

The relic density is a strong constraint, since it is a positive measurement with a rather small experimental uncertainty. Therefore, the shape and size of the 2D 95\% confidence regions presented in this section will be determined predominantly by the relic density as measured by PLANCK.

The color code in the $95 \%$ confidence region of Fig. [5](a) shows the composition of the lightest neutralino, which is the lightest mass eigenstate of mixed bino, wino, up-type higgsino and downtype higgsino gauge eigenstates,

$$
\chi \equiv \chi_{1}^{0}=Z_{1} \tilde{B}+Z_{2} \tilde{W}+Z_{3} \tilde{H}_{u}+Z_{4} \tilde{H}_{d},
$$

where the coefficients $Z_{i}(i \leq 4)$ are determined by diagonalizing the neutralino mass matrix. To 


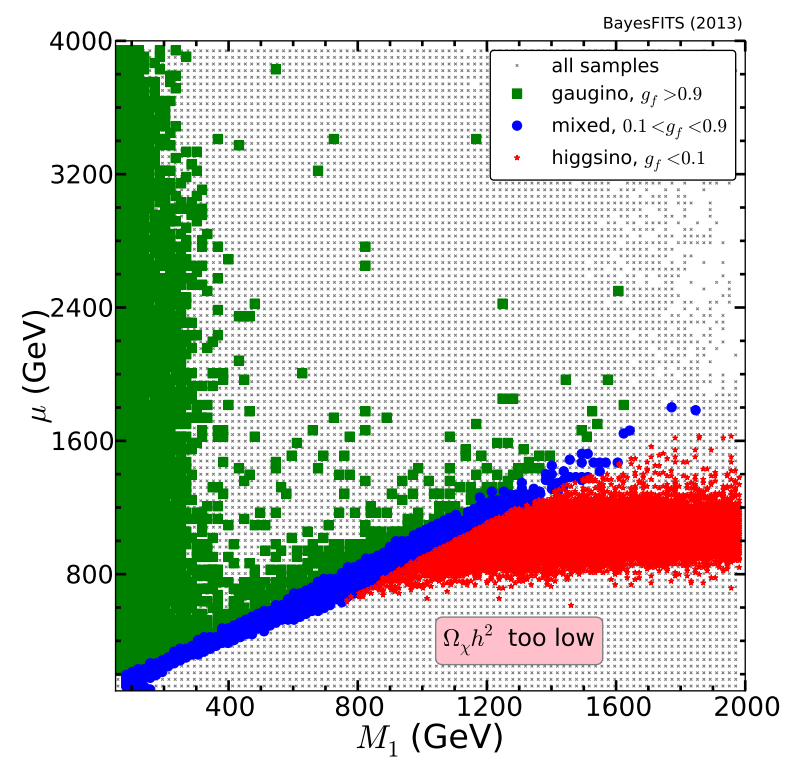

(a)

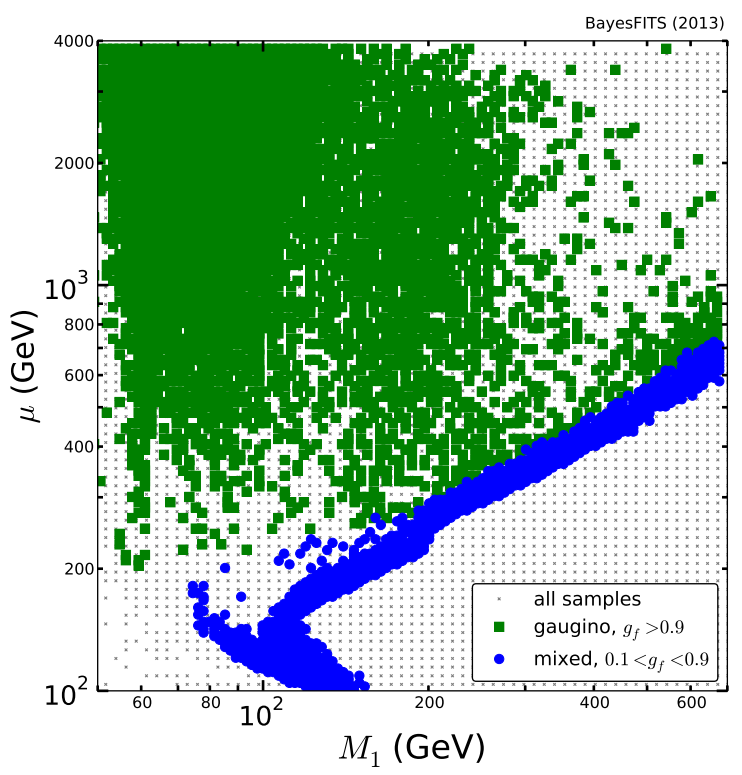

(b)

Figure 5: (a) Scatter plot of p9MSSM points in the $\left(M_{1}, \mu\right)$ plane. Gray crosses show the points excluded by the basic likelihood at the $95 \%$ C.L. Allowed points are divided by the composition of the neutralino: gaugino-like (green squares), mixed (blue circles), or higgsino-like (red stars). (b) Magnified plot of the low-mass region of (a) with logarithmic scales.

describe the neutralino compositions, it is convenient to introduce a gaugino fraction, $g_{f}=Z_{1}^{2}+Z_{2}^{2}$. When $g_{f}$ is close to 1 , gauginos dominate the neutralino; on the other hand the neutralino will be higgsino-like if $g_{f} \simeq 0$. The points for which the neutralino is a nearly pure gaugino are presented as green squares, the points for which the neutralino is higgsino-like are marked as red stars, and the points for which the neutralino composition is some mixture of gaugino and higgsino states are shown as blue circles.

A bino-like LSP is obtained when $m_{\chi} \approx M_{1}<\mu$. One can see in Fig. 5. (a) that, when $M_{1} \lesssim$ $400 \mathrm{GeV}$, gaugino-like neutralino DM satisfies the relic abundance for a broad range of $\mu$ values (green squares on the left). In Fig. 馬(b) we show a zoomed-in view of this region of the parameter space. One can identify two separate gaugino-like branches. On the left, for $M_{1} \lesssim 65 \mathrm{GeV}$ the correct value of the relic abundance is obtained by efficient annihilation to $b$-quarks in the early universe through $s$-channel diagram exchange of the lightest Higgs boson (note that for this to occur the neutralino must have a nonzero higgsino component) and, for slightly larger $M_{1} \lesssim 100 \mathrm{GeV}$, through "bulk" annihilation to leptons through $t$-channel slepton exchange. We will refer to this region as the $h$-resonance/bulk region [117] (HR/bulk). To the right of the HR/bulk region, for $100-150 \mathrm{GeV} \lesssim M_{1} \lesssim 300 \mathrm{GeV}$, a second area for gaugino DM can be observed, where the correct relic density is obtained through coannihilation with sleptons of the three generations [118] (selectrons and smuons for $100 \mathrm{GeV} \lesssim M_{1} \lesssim 200 \mathrm{GeV}$, staus for $200 \mathrm{GeV} \lesssim M_{1} \lesssim 300 \mathrm{GeV}$ ). We will refer to this region as the slepton-coannihilation (SC) region.

By decreasing $\mu$ down to $\mu \approx M_{1}\left(<M_{2}\right)$, the higgsino fraction in the neutralino increases (mixed composition, blue circles). Along the blue strip that extends to $\mu \approx M_{1} \lesssim 800 \mathrm{GeV}$ in Fig. [5(a), the relic density constraint is satisfied thanks to $\chi \chi$ annihilation into gauge bosons, 


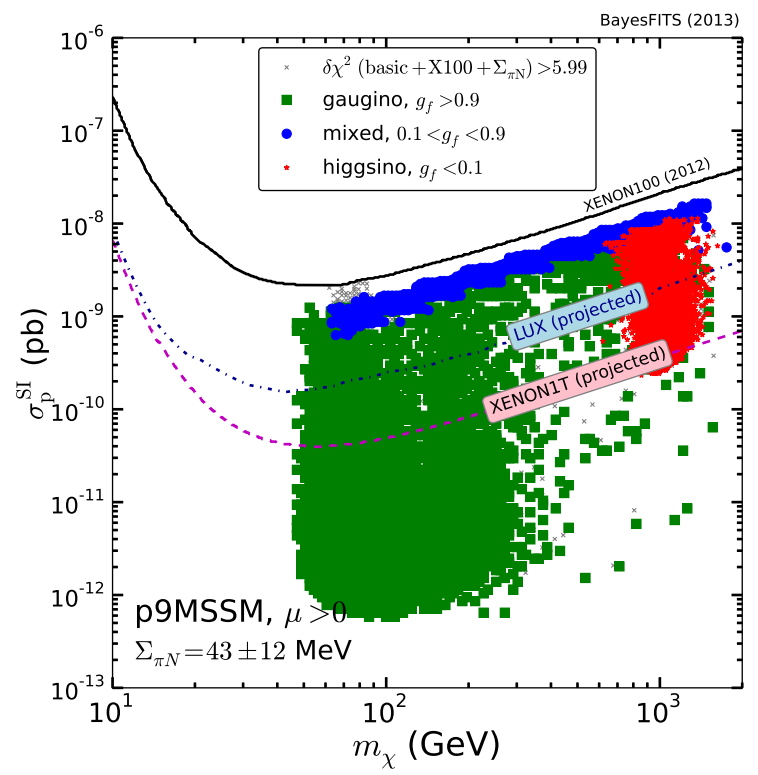

(a)

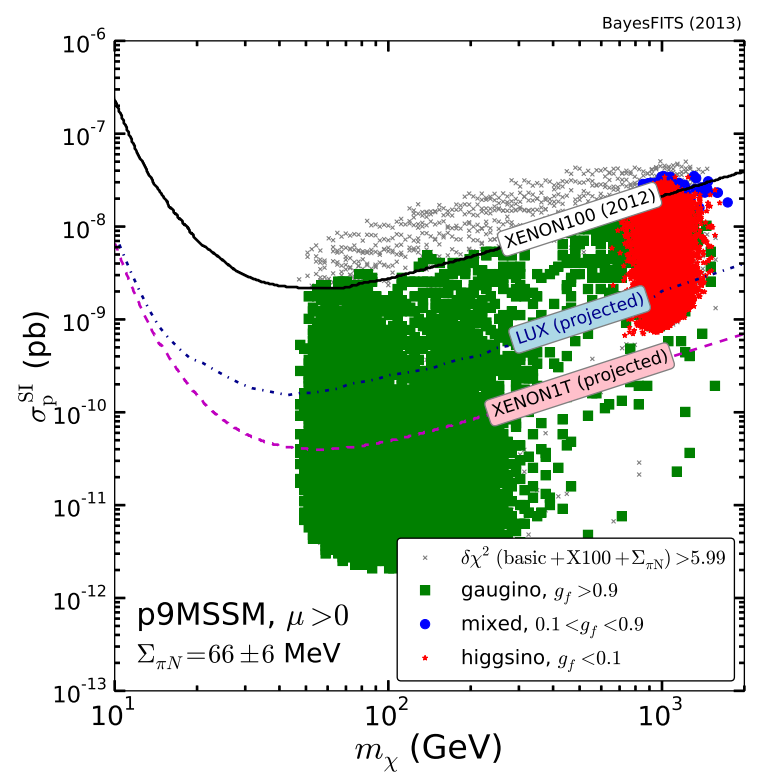

(b)

Figure 6: p9MSSM points that are allowed at $2 \sigma$ by the basic constraints in the $\left(m_{\chi}, \sigma_{p}^{\mathrm{SI}}\right)$ plane. The points consistent at $2 \sigma$ with the basic and XENON100 constraints are divided by the composition of the neutralino: gaugino-like (green squares), mixed (blue circles), or higgsino-like (red stars). Points excluded at the 95\% C.L. by basic+XENON100 are shown as gray crosses. (a) $\Sigma_{\pi N}=43 \pm 12 \mathrm{MeV},(\mathrm{b}) \Sigma_{\pi N}=66 \pm 6 \mathrm{MeV}$.

through $t$-channel exchange of higgsino-like $\chi_{1}^{ \pm}$and/or $\chi_{2}^{0}$. This is the p9MSSM equivalent of the focus point/hyperbolic branch (FP/HB) region of the CMSSM [119, 120], and we will loosely use the same acronym to describe this region of the p9MSSM in what follows. The "hook" feature in points with mixed composition at $\mu \lesssim 150 \mathrm{GeV}$ results from a $W W$ threshold. In only that region, the neutralino has a bino/higgsino composition and, at the same time, $m_{\chi}<M_{W}$ while $m_{\chi_{1}^{ \pm}}>94 \mathrm{GeV}$. Because the $\chi \chi \rightarrow W^{+} W^{-}$annihilation is suppressed by a threshold due to chargino exchange, the relic density is not too small. Note that the green strip of gaugino-like DM, adjacent and above the $\mathrm{FP} / \mathrm{HB}$ region up to $M_{1} \simeq 1.2-1.6 \mathrm{TeV}$ in Fig. [5.(a) is the AF region [121].

As one considers ever larger $\mu$ along the $\mathrm{FP} / \mathrm{HB}$ region, the neutralino becomes almost purely higgsino-like, and its mass stabilizes at $m_{\chi} \approx \mu \simeq 1 \mathrm{TeV}$. We call this the $1 \mathrm{TH}$ region and it is indicated with red stars in Fig. 馬(a). Here $\chi$ and $\chi_{2}^{0}$ are either both higgsino-like or one of them is higgsino- and the other bino-like, respectively, while $\chi_{1}^{ \pm}$is always higgsino-like. The relic density constraint is satisfied for broad ranges of $M_{1}$, partially through LSP co-annihilation with the second lightest neutralino, $\chi_{2}^{0}$, and/or the lightest chargino $\chi_{1}^{ \pm}$.

Note, finally, the lower density of gray dots in the lower left corner of Fig. 馬(b), In fact, in that region of the parameter space the LEP constraints on the chargino mass, Eq. (2), become much harder to satisfy.

\subsection{Impact of the XENON100 limit}

In this subsection we analyze the impact of the XENON100 90\% C.L. upper bound on the parameter space of the p9MSSM. We emphasize that the bound is applied through the likelihood function 
given by Eq. (8).

In Figs. G(a) and G(b), we show the difference between the basic $95 \%$ confidence region (all points) and the 95\% confidence region obtained by adding the likelihood of Eq. (8) (all points except gray crosses $)$ in the $\left(m_{\chi}, \sigma_{p}^{\mathrm{SI}}\right)$ plane. The color code describes the gaugino fraction of the LSP, and it is the same as in Fig. 5 .

In Fig. G(a), we show the case with nuclear physics uncertainties parametrized around $\Sigma_{\pi N}=$ $43 \pm 12 \mathrm{MeV}$, as described in Sec. 3.2. One can see that a small fraction of points characterized by mixed gaugino-higgsino composition and $m_{\chi} \simeq 60-90 \mathrm{GeV}$ is excluded at the $95 \%$ C.L. by the global likelihood. For these points, in fact, the lightest Higgs boson exchange in the $t$ channel due to the non-negligible higgsino fraction of the neutralino can enhance $\sigma_{p}^{\mathrm{SI}}$. At the tree level, there are only two Feynman diagrams contributing to $\sigma_{p}^{\mathrm{SI}}[122$; $t$-channel diagram Higgs exchange and $s$-channel squark resonance. While the squark resonance is suppressed by the fact that LHC limits now imply heavy squarks, one can always tune the gaugino fraction to increase or decrease the contribution from the Higgs exchange mode. Also, the mass values for the excluded points correspond to the region of greater sensitivity for XENON100.

Note that, as pointed out in Sec. 3.2, the large theoretical uncertainties drastically reduce the impact of the XENON100 constraint on the parameter space. This point is emphasized in Fig. G(b), where we parametrize the theoretical uncertainties around $\Sigma_{\pi N}=66 \pm 6 \mathrm{MeV}$, following the determination of [113, 115]. One can see that, in this case, the mixed gaugino/higgsino region of the parameter space becomes almost entirely excluded at the 95\% C.L.

In Figs. G(a) and G(b) we also plot the expected reaches of LUX [123] and XENON1T [124]. One can see that, even when substantial theoretical uncertainties are taken into account, those experiments have the potential to bite into a large fraction of the mixed gaugino/higgsino region, particularly if the determination of $\Sigma_{\pi N}$ stabilizes in the future around the larger value.

\subsection{Impact of $\delta(g-2)_{\mu}$ and limits from the LHC}

As mentioned in Sec. 2, we imposed a GUT-inspired universality condition, $M_{1}=0.5 M_{2}$, on our parameter space. We additionally assumed $m_{\tilde{L}_{1,2}}=M_{1}+50 \mathrm{GeV}$, in order to enhance $(g-2)_{\mu}$.

The anomalous magnetic moment of the muon, $(g-2)_{\mu}$, is subject to SUSY contributions that can enhance its SM value for $\mu>0$. At the leading order, the dominant terms are given by a chargino-sneutrino (of the second generation) loop and by a neutralino-smuon loop (Ref. [59] and references therein).

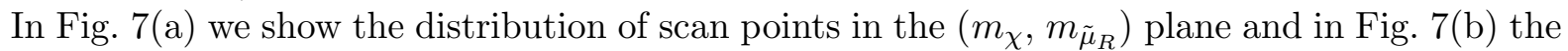
one in the $\left(m_{\tilde{\nu}_{\mu}}, m_{\chi_{1}^{ \pm}}\right)$plane. The purple (orange) squares (circles) indicate the points for which $\delta(g-2)_{\mu}$ is satisfied at $1 \sigma(2 \sigma)$, i.e., $\chi_{g-2}^{2}<1\left(\chi_{g-2}^{2}<4\right)$.

For $\mu \ll M_{2}=2 M_{1}, \chi$ and $\chi_{1}^{ \pm}$are higgsino-like, or $\chi$ is a bino/higgsino mixed state and $\chi_{1}^{ \pm}$ is higgsino-like, and their masses are comparable. They are both lighter than the sleptons, so $\delta(g-2)_{\mu}$ gives the bound $m_{\tilde{\mu}} \simeq m_{\tilde{\nu}_{\mu}} \lesssim 600 \mathrm{GeV}$, which is independent of our parametrization. On the other hand, for $\mu>M_{2}$, the neutralino is bino-like and the (wino-like) chargino mass presents an upper limit $m_{\chi_{1}^{ \pm}} \approx 2 m_{\chi}$. Thus, by placing an upper bound on the mass of the neutralino, $m_{\chi} \lesssim 500 \mathrm{GeV}$, the $(g-2)_{\mu}$ constraint indirectly places a limit on the chargino mass $m_{\chi_{1}^{ \pm}} \lesssim 1 \mathrm{TeV}$. Besides, since we have set $m_{\tilde{L}_{1,2}}=M_{1}+50 \mathrm{GeV}$, when the neutralino is bino-like an upper bound on its mass translates on an indirect upper bound on the smuon mass.

So, the two parameters of relevance to the $(g-2)_{\mu}$ constraint are $\mu$ and $M_{2}$. On the other hand, when the relic density constraint is included, the region where the neutralino is almost purely higgsino-like is excluded at the $95 \%$ C.L. for $m_{\chi} \ll 1 \mathrm{TeV}$, as shown in Figs. 5 and 6 . In Figs. 耼) 


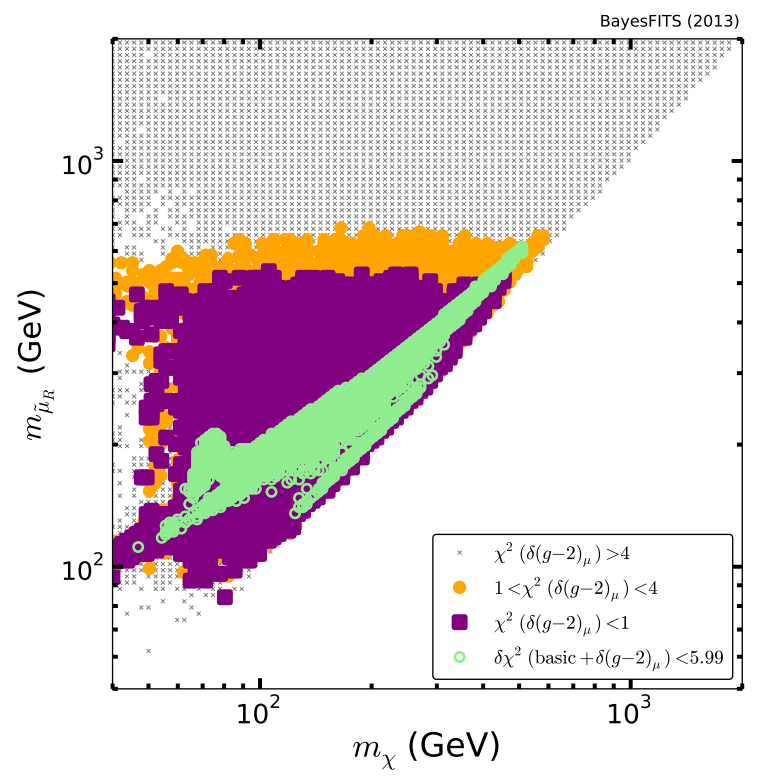

(a)

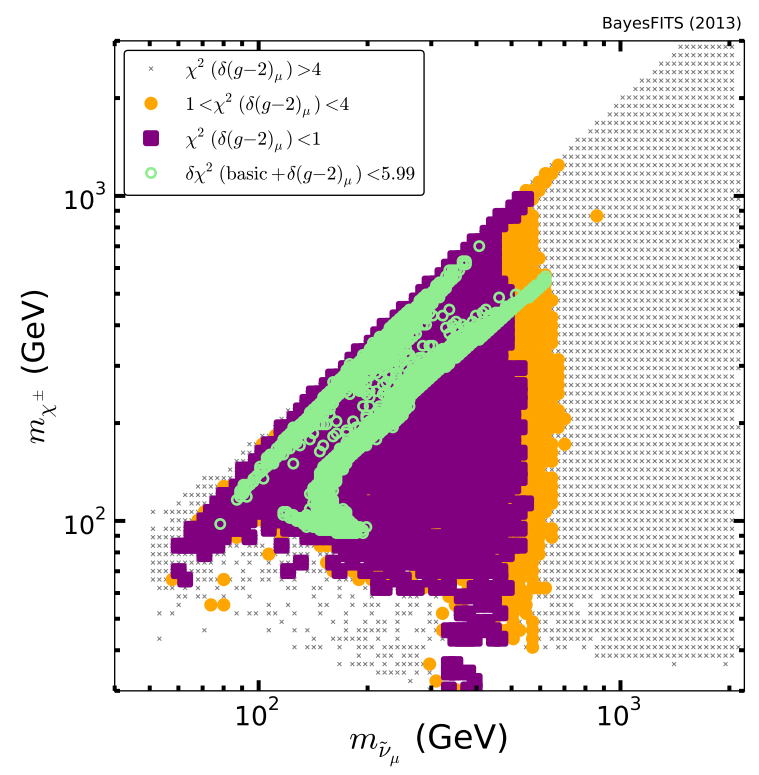

(b)

Figure 7: (a) p9MSSM points scattered in the $\left(m_{\chi}, m_{\tilde{\mu}}\right)$ plane. Points with a $\chi^{2}$ from the $(g-2)_{\mu}$ constraint of greater than 4 are shown with gray crosses. Points with $\chi^{2}<1$ and $1<\chi^{2}<4$ are shown with purple squares and orange circles, respectively. Points compatible with both $(g-2)_{\mu}$ and basic at $2 \sigma$ are shown with light green circles. (b) p9MSSM points scattered in the $\left(m_{\tilde{\nu}_{\mu}}\right.$, $\left.m_{\chi_{1}^{ \pm}}\right)$plane. The color code is the same as in (a).

and $7(\mathrm{~b})$, the points for which the basic set of constraints is satisfied together with $\delta(g-2)_{\mu}$ at $2 \sigma$ are shown as green empty circles. One can identify two main branches: on the diagonal, close to the edge of the parameter space, $\mu>M_{2}$; elsewhere $\mu \leq M_{2}$.

Inclusive searches for SUSY particles, like the $\alpha_{T}$ search that we included in the likelihood function, have little sensitivity to models in which the squarks are heavy and the sleptons are light. On the other hand, searches for EW production of pair-produced charginos with multiple leptons and missing energy in the final state are designed to probe the parameter space of the theory that overlaps with the $(g-2)_{\mu}$ sector. As described in Sec. 3.1, we calculated the likelihood for the CMS EW-production search at $\sqrt{s}=8 \mathrm{TeV}$ and $\mathcal{L}=9.2 / \mathrm{fb}$, which is the one giving the strongest limits. Due to the great number of points in our scan that can be affected by this search, we apply the numerical procedure to calculate the likelihood to a randomly selected sample of approximately 40,000 points, all of which satisfy the basic set of constraint at the $95 \%$ C.L.

We show the exclusion due to EW production in Fig. 8, where we plot the $95 \%$ confidence region for the basic constraints applied to the thinned chain in the $\left(m_{\chi}, \delta(g-2)_{\mu}\right)$ plane (note that after the other constraints are taken into account, in the p9MSSM $\delta(g-2)_{\mu}$ is parametrized only by $\left.m_{\chi}\right)$. The color code is the same as in Figs. 1 and 2 , The cyan circles are excluded at the $95.0 \%$ C.L., and the gray dots at the $99.7 \%$ C.L. One can see again that the $(g-2)_{\mu^{-}} 2 \sigma$ region requires $m_{\chi} \lesssim 500 \mathrm{GeV}$, whereas the strongest EW-production search at the LHC requires $m_{\chi} \gtrsim 200-250 \mathrm{GeV}$, the range depending on the parameters and on the chargino and neutralino compositions. Thus, there is a window of availability in the parameter space for $200 \mathrm{GeV} \lesssim m_{\chi} \lesssim$ $500 \mathrm{GeV}$, of points in good agreement with all constraints. 


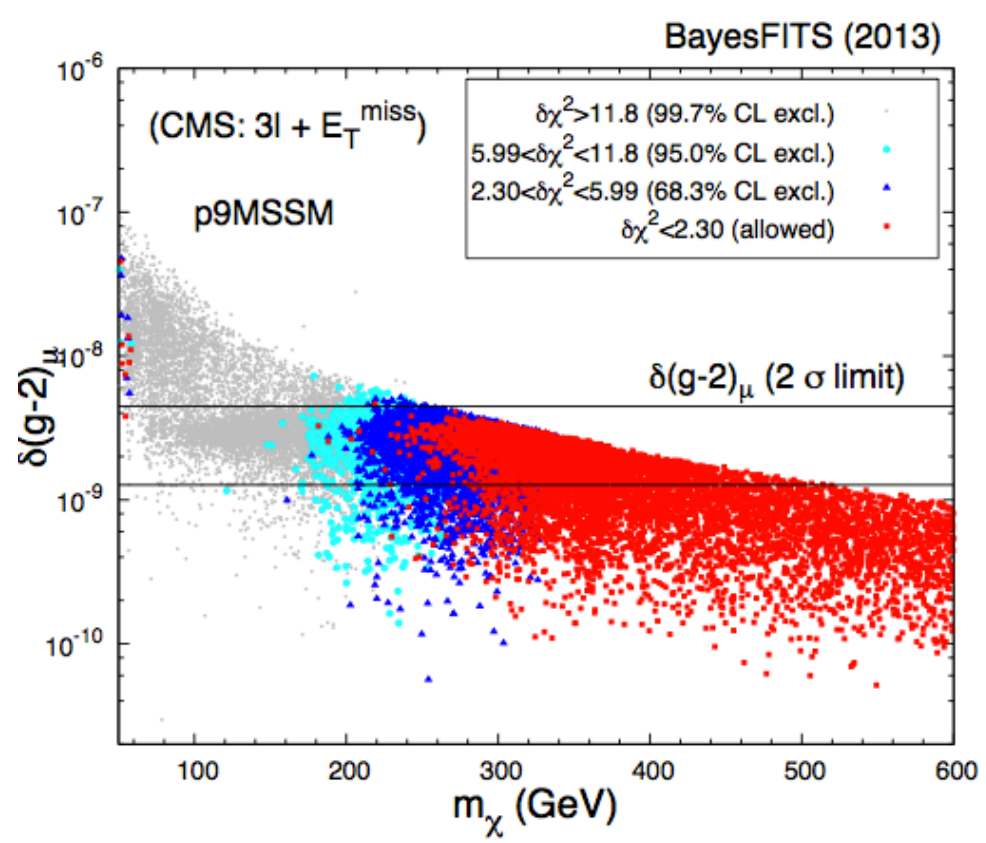

Figure 8: Our chargino-neutralino pair production (EW) likelihood in the $\left.\left(m_{\chi}, \delta(g-2)\right)_{\mu}\right)$ plane for a thinned sample of p9MSSM points consistent at $2 \sigma$ with the basic constraints. The allowed $2 \sigma$ interval for $(g-2)_{\mu}$ is shown with horizontal solid lines. The $\delta \chi^{2}$ from EW production is indicated by the different colors: grey dots, cyan circles and blue triangles are excluded at the $99.7 \%, 95 \%$ and $68.3 \%$ C.L., respectively.

As a side note, on the left of Fig. 8 one can notice the presence of some points at $m_{\chi} \simeq 50 \mathrm{GeV}$ not excluded by the LHC EW production search. Those points are characterized by $\left|m_{\chi_{2}^{0}\left(\chi_{1}^{ \pm}\right)}-m_{\tilde{l}}\right| \lesssim$ $1 \mathrm{GeV}$ (the neutralino is bino-like and we chose $m_{\tilde{L}_{1,2}}=M_{1}+50 \mathrm{GeV}$ and $M_{1}=0.5 M_{2}$ ) and the intermediate sleptons are considered on-shell by PYTHIA. As a consequence, two of the final state leptons are soft and the search loses sensitivity.

\subsection{Higgs mass and $h \rightarrow \gamma \gamma$ signal rate}

In Fig. $9(\mathrm{a})$, we present the $1 \sigma$ (blue points) and $2 \sigma$ (cyan diamonds) confidence regions of the basic likelihood in the $\left(m_{h}, m_{\tilde{t}_{1}}\right)$ plane. One can see that $m_{h}$ presents a normal distribution around the central value, as expected from the Gaussian likelihood. A Higgs mass consistent at $1 \sigma$ with all constraints including the measured value of the Higgs mass can be obtained for stop masses as small as $\sim 600 \mathrm{GeV}$, thanks to maximal stop mixing, $\left|X_{t}\right| / M_{\mathrm{SUSY}} \simeq \sqrt{6}$. The $2 \sigma$ region allows for stop masses as small as $\sim 280 \mathrm{GeV}$. For $m_{\chi} \lesssim 200 \mathrm{GeV}$ the $\alpha_{T}$ constraint excludes $m_{\tilde{t}_{1}} \lesssim 350 \mathrm{GeV}$ at the 95\% C.L. (we remind the reader that our implementation of the $\alpha_{T}$ search is very conservative for the third generation squarks, as was discussed in Sec. 3.1) unless the SUSY spectrum is compressed $\left(m_{\chi} \gtrsim m_{\tilde{t}_{1}}-M_{t}\right)$, in which case the search becomes sensitive to initial state radiation, and a reliable bound cannot be produced. This is the case for the two points at $m_{\tilde{t}_{1}} \lesssim 350 \mathrm{GeV}$ shown in the plot, which are characterized by neutralino masses of order $m_{\chi} \sim 220-230 \mathrm{GeV}$.

In this subsection we also check to what extent the MSSM lightest Higgs boson $h$ complies with the LHC observations in the $\gamma \gamma$ channel. In order to do so, we calculate its reduced cross section, 


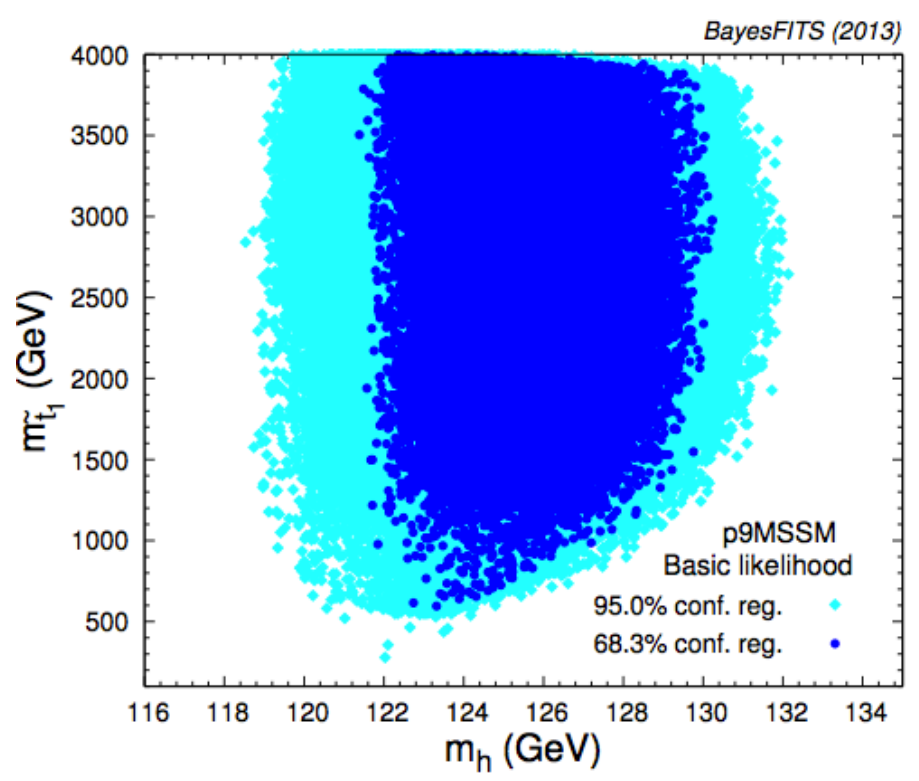

(a)

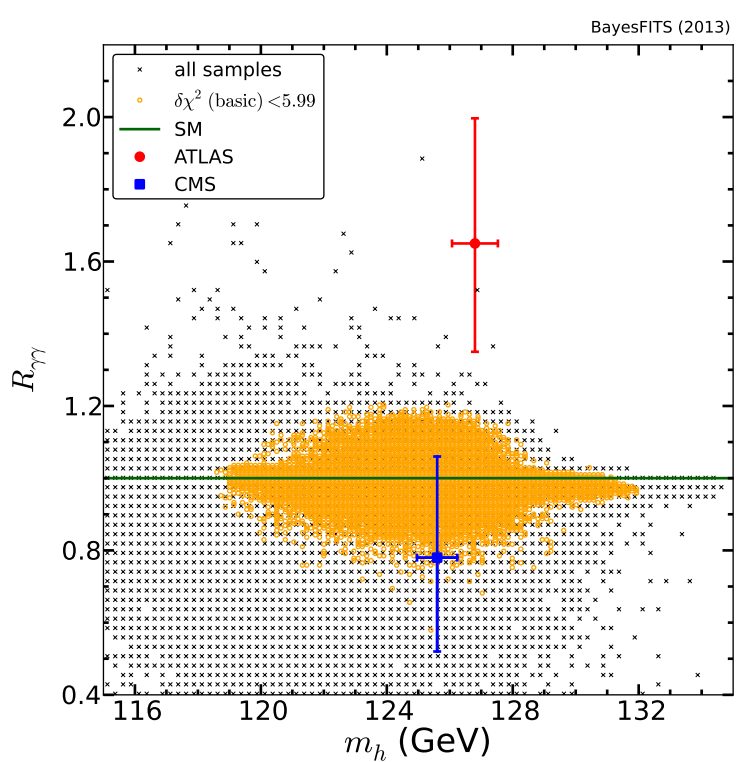

(b)

Figure 9: (a) 68.3\% (blue points) and 95.0\% (cyan diamonds) confidence regions for the basic likelihood in the $\left(m_{h}, m_{\tilde{t}_{1}}\right)$ plane. (b) Scatter plot of p9MSSM points in the $\left(m_{h}, R_{\gamma \gamma}\right)$ plane. The points consistent with basic constraints at $2 \sigma$ are shown as orange circles. The Standard Model value $R_{\gamma \gamma}=1$ is marked with a horizontal solid line. The ATLAS and CMS measurements are marked with red and blue cross hairs respectively.

defined in literature (see, e.g., [125]) as

$$
R_{h}(\gamma \gamma)=\frac{\sigma(p p \rightarrow h)}{\sigma\left(p p \rightarrow h_{\mathrm{SM}}\right)} \times \frac{B R(h \rightarrow \gamma \gamma)}{B R\left(h_{\mathrm{SM}} \rightarrow \gamma \gamma\right)}
$$

The branching ratios in Eq. (10) are calculated using FeynHiggs 2.9.4 [79, 80, 81, 82] both for the MSSM $h$ and the SM Higgs, $h_{\mathrm{SM}}$, with the same mass. The total Higgs production cross sections are computed from the parton level production cross sections as follows:

$$
\frac{\sigma(p p \rightarrow h)}{\sigma\left(p p \rightarrow h_{\mathrm{SM}}\right)}=\sum_{Y \in \operatorname{prod}} \frac{\sigma\left(p p \rightarrow Y \rightarrow h_{\mathrm{SM}}\right)}{\sigma\left(p p \rightarrow h_{\mathrm{SM}}\right)} \times \frac{\sigma(Y \rightarrow h)}{\sigma\left(Y \rightarrow h_{\mathrm{SM}}\right)},
$$

where $Y$ spans over the different production channels: gluon-fusion, vector boson-fusion, Higgsstrahlung off a $W / Z$ boson, and associated Higgs production with top quarks. The parton level cross sections $\sigma(Y \rightarrow h)$ and $\sigma\left(Y \rightarrow h_{\mathrm{SM}}\right)$ are computed for $h$ and $h_{\mathrm{SM}}$ using FeynHiggs 2.9.4. For each production mode $Y$, we then obtain a coefficient $\sigma\left(p p \rightarrow Y \rightarrow h_{\mathrm{SM}}\right) / \sigma\left(p p \rightarrow h_{\mathrm{SM}}\right)$ to transform the calculation from the parton to the hadron level using the public tables provided by the LHC Higgs Cross Section Working Group [126, 127] for $\sqrt{s}=8 \mathrm{TeV}$.

In Fig. $9(\mathrm{~b})$ we show the p9MSSM distribution of points in the $\left(m_{h}, R_{h}(\gamma \gamma)\right)$ plane. The complete sample is shown in gray crosses, while the points that satisfy the basic constraints at $2 \sigma$ are shown as orange circles. We also superimpose the CMS and ATLAS central values and experimental errors. One can notice the the $\gamma \gamma$ rate can be enhanced in the p9MSSM by $20 \%$ with respect to the SM, when all constraints are taken into account. The value of $R_{h}(\gamma \gamma)$ can, however, 


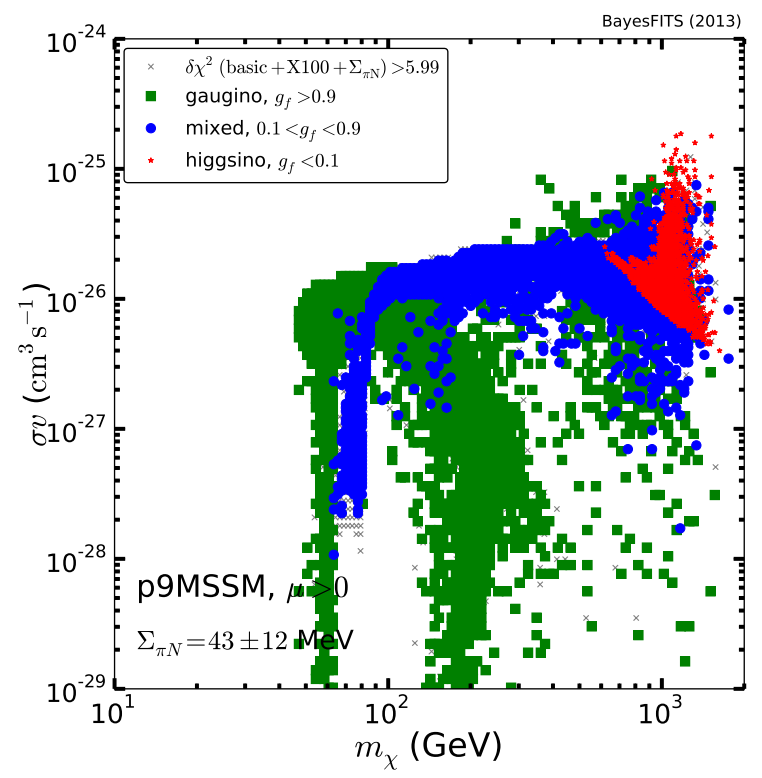

(a)

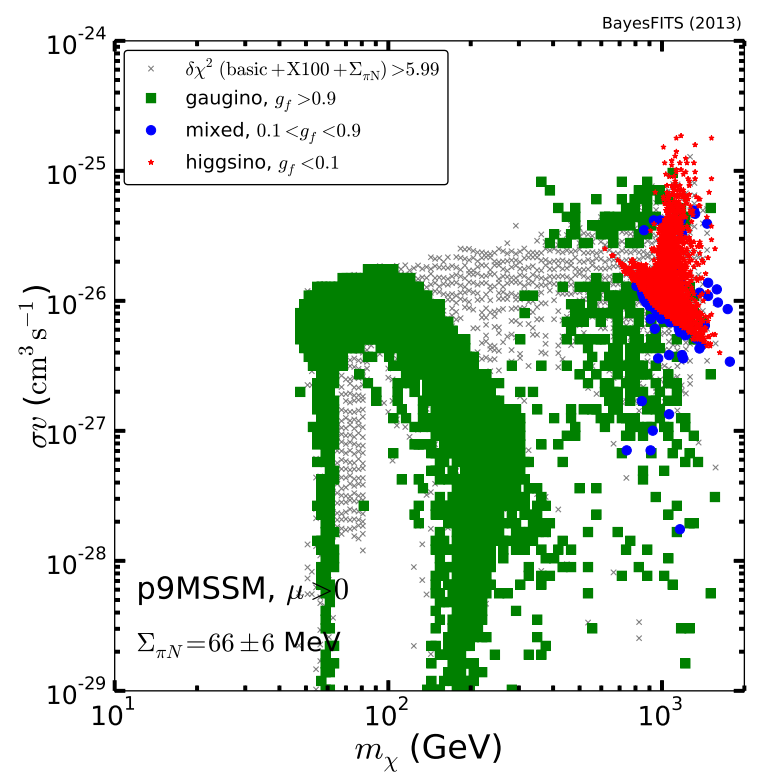

(b)

Figure 10: p9MSSM points allowed at $2 \sigma$ by the basic constraints in the $\left(m_{\chi}, \sigma v\right)$ plane. The points consistent at $2 \sigma$ with the basic and XENON100 constraints are shown for different composition of the neutralino: gaugino-like (green squares), mixed (blue circles), or higgsino-like (red stars). (a) $\Sigma_{\pi N}=43 \pm 12 \mathrm{MeV},(\mathrm{b}) \Sigma_{\pi N}=66 \pm 6 \mathrm{MeV}$.

also be as low as 0.6. Different mechanism of di-photon rate enhancement were discussed in the literature, including the effects of the light staus [13] or light charginos [128]. In our scan, di-photon rate enhancement is in general a combination of different mechanisms.

\subsection{Indirect detection of DM}

Having tested the compatibility of our model with the limits from XENON100, $(g-2)_{\mu}$ and the LHC SUSY searches, we now proceed to examine the implications from ID of DM experiments on the allowed regions of the parameter space. We derive constraints from Fermi $\gamma$-ray data from the GC of the Milky Way as well as from its dSphs and from IceCube data on neutrinos from the Sun.

The quantity relevant for indirect DM detection searches is the neutralino annihilation cross section in the limit of small momenta, $\left.\sigma v \equiv \sigma v\right|_{p \rightarrow 0}$. In Fig. 10 we present the $95 \%$ confidence regions obtained by adding the XENON100 likelihood to the basic set of constraints, projected onto the $\left(m_{\chi}, \sigma v\right)$ plane. As was done in Fig. 6, we show the case with $\Sigma_{\pi N}=43 \pm 12 \mathrm{MeV}$ in Fig. 10 (a) and the one with $\Sigma_{\pi N}=66 \pm 6 \mathrm{MeV}$ in Fig. 10(b). The color code describing the gaugino fraction of the LSP is the same as in the previous figures.

Different mechanisms of generating the correct value of the relic density, associated with different regions of the $\left(M_{1}, \mu\right)$ plane in Fig. 5, can be also identified in Fig. 10(a), The first vertical branch on the left, characterized by gaugino-like neutralinos at $m_{\chi} \simeq 60 \mathrm{GeV}$, corresponds to the $\mathrm{HR}$ region of the $\left(M_{1}, \mu\right)$ plane, while the adjacent gaugino region at $\sigma v \simeq 10^{-26} \mathrm{~cm}^{3} \mathrm{~s}^{-1}$ corresponds to the bulk region.

The second vertical branch at $m_{\chi} \simeq 80 \mathrm{GeV}$, with mixed gaugino/higgsino composition, becomes horizontal for larger masses and extends to $\sim 800 \mathrm{GeV}$. As we pointed out while discussing 
Fig. 5, this is the MSSM counterpart of the FP/HB region in GUT-constrained models. One can see that the opening of the $W W$ annihilation channel at $m_{\chi} \gtrsim 80 \mathrm{GeV}$ increases the value of $\sigma v$. Also note that the only points excluded at the 95\% C.L. by adding XENON100 in the case $\Sigma_{\pi N}=43 \pm 12 \mathrm{MeV}$ lie in this region (gray crosses).

More to the right, there is an additional vertical branch, at $m_{\chi} \simeq 100-300 \mathrm{GeV}$, characterized by a large gaugino fraction. This is the SC region. The AF region can instead be identified with the widespread area of gaugino-like LSP at $400 \mathrm{GeV} \lesssim m_{\chi} \lesssim 1.2 \mathrm{TeV}$.

In the $1 \mathrm{TH}$ region, for $800 \mathrm{GeV} \lesssim m_{\chi} \lesssim 1.2 \mathrm{TeV}$, about $50 \%$ of the contribution to the relic density reduction comes from $\chi_{1} \chi^{ \pm}$and $\chi_{1} \chi_{2}$ coannihilations. Most of the remaining half of the total contribution in this branch is due to $\chi \chi$ annihilation to fermions.

We remind the reader that, although the value of $\sigma v$ is related to the value of the relic abundance of neutralinos at present, the correlation between the two quantities is not straightforward, since the annihilation (and coannihilation) cross sections used to determine the relic density are thermally averaged in the early Universe. This is the reason why many of the points in Fig. 10 present cross sections in excess of or below the "default" value of $3 \times 10^{-26} \mathrm{~cm}^{3} / \mathrm{s}$ for the relic density. We give a short review of this issue in Appendix B.

\subsection{1 $\gamma$ rays from dSphs}

In order to quantify the impact of the Fermi-LAT exclusion bounds from dSphs in the $\left(m_{\chi}, \sigma v\right)$ plane of the p9MSSM we use a publicly available code written by one of us [129], which calculates the likelihood function for each model point given the 4-year data made available by the experimental collaboration [130]. The program, described in detail in [129], takes the $\gamma$-ray yield spectrum for $\chi \chi$ annihilation at each model point, $d N_{\gamma} / d E_{\gamma}$, as an input.

The likelihood does not exclude any of the points in our scan. One should bear in mind, though, that the values of $d N_{\gamma} / d E_{\gamma}$ given by different simulation codes such as PYTHIA6 [99], PYTHIA8 [131, or Herwig++ [132] can differ by a significant factor [133, hence the predictions are subject to a certain amount of theoretical uncertainty. We incorporated possible uncertainties in our results by multiplying the SUSY-predicted $\gamma$-ray flux with a phenomenological boost factor, $B_{F}$, adjusted point by point to maximize the likelihood. We show in Fig. 11|(a) the points of the $95 \%$ confidence region in the $\left(m_{\chi}, \sigma v\right)$ plane from the combined basic+XENON100 profile-likelihood (we assume $\Sigma_{\pi N}=43 \pm 12 \mathrm{MeV}$ ). The color code indicates the boost factor required at each point to maximize the dSphs likelihood [129]. For the green diamonds, $B_{F}<10$, for the orange squares $10<B_{F}<20$, and for the gray circles $B_{F}>20$. One can see that the dSphs 4-year data can be used to test some of the points (green points) not tested by XENON100 (see Fig. 10|(a) for comparison), provided a small boost factor is assumed.

\subsection{2 $\gamma$ rays from the Galactic Center}

Of the points shown in green in Fig. 11|(a), we take the three with the smallest $B_{F}$ and we simulate numerically the diffused $\gamma$-ray fluxes from the GC with the GALPROP package [134]. The p9MSSM parameters of the three selected points are given in Table 3. We assume the Einasto halo profile [135] and a local density $\rho_{0}=0.4 \mathrm{GeV} \mathrm{cm}^{-3}\left[136\right.$. The $\gamma$ rays from the GC can be produced via $\pi^{0}$ decay, as well as inverse Compton scattering and bremsstrahlung of the electrons and positrons along the propagation line in the interstellar medium. For each point, we insert $d N_{\gamma} / d E_{\gamma}$ into GALPROP to calculate $\gamma$-ray fluxes from $\pi^{0}$ decay. For inverse Compton scattering and bremsstrahlung, we insert $d N_{e^{ \pm}} / d E_{e^{ \pm}}$into GALPROP to obtain $\gamma$-ray fluxes due to the propagation of the $e^{ \pm}$from the GC to the Earth. In our analysis, we use the best-fit propagation model [137]. In Fig. 11](b), we show the 


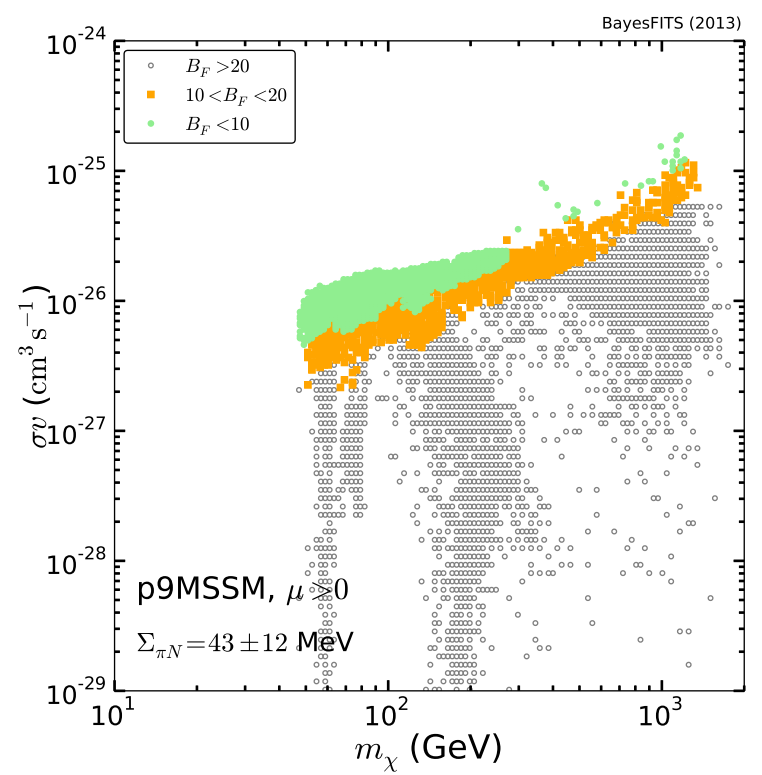

(a)

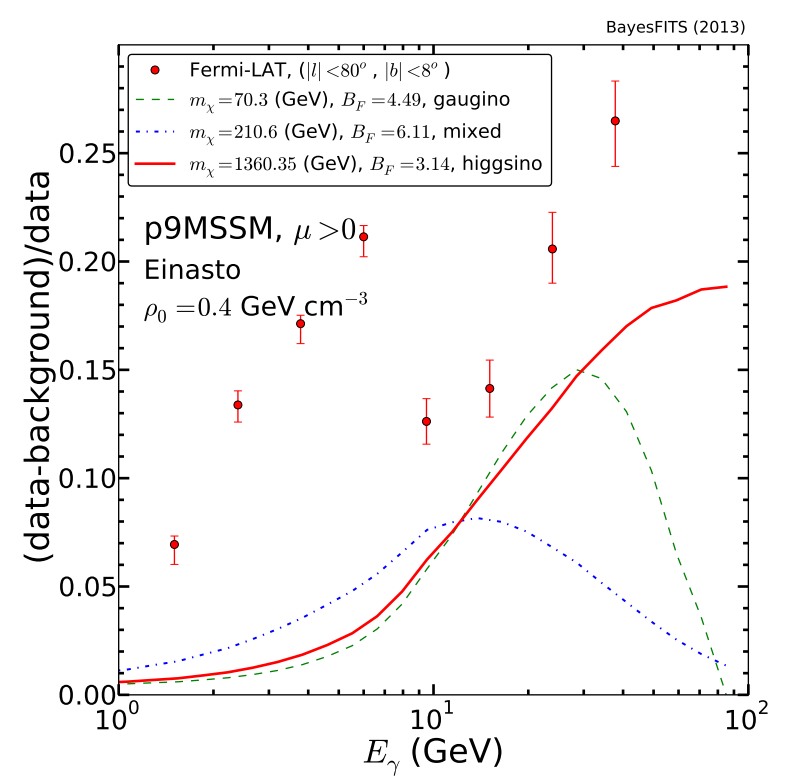

(b)

Figure 11: (a) p9MSSM points consistent with the basic and XENON100 constraints at $2 \sigma$ in the $\left(m_{\chi}, \sigma v\right)$ plane. The $\gamma$-ray flux boost factor $B_{F}$ is indicated by the different colors: points with $B_{F}>20$ are shown with gray circles, $10<B_{F}<20$ with orange squares, and $B_{F}<10$ with green diamonds. (b) The expected $\gamma$-ray flux distribution above the background from the GC for three p9MSSM points (shown in Table 3) with small $B_{F}$, but with different neutralino composition: a point with gaugino-like neutralinos is shown with a dashed green line, mixed neutralinos with a dash-dotted blue line, and higgsino-like neutralinos with a solid red line. The Einasto halo profile and a local density of $\rho_{0}=0.4 \mathrm{GeVcm}^{-3}$ have been assumed. The measured $\gamma$-ray flux distribution from Fermi-LAT is shown with red circles.

comparison of the residual "data-background/data" ratio, calculated for the three selected points in the Galactic coordinates $|l|<80^{\circ}$ and $|b|<8^{\circ}$, with the experimental data published in [138]. Interestingly, the points that seem to be closer to reproducing the data (with $B_{F}<5$ ) are the ones characterized either by heavy higgsino-like neutralinos, because of the enhancement in $\sigma v$ and of a substantial integrated flux, or by a light gaugino-like $\chi$, because the flux suppression due to $m_{\chi}^{2}$ is reduced. A more refined analysis of the DM halo and astrophysical background sources will, however, be required to draw more quantitative conclusions.

\subsubsection{Sensitivity of IceCube}

As mentioned earlier, data from the IceCube neutrino telescope can also be used to test the parameter space of the model. In particular, neutrinos from the Sun provide a means to test the spin-dependent cross section of neutralino-nucleon elastic scattering, $\sigma_{p}^{\mathrm{SD}}$, to which DD experiments are insensitive [105].

We use DarkSUSY to compute the 5-year, 95\% C.L. sensitivity of the IceCube's 86 string configuration. We adopt the default values of the effective area, of the atmospheric neutrino background, and of the quark spin content of the nucleon. The maximum opening angle is set to $20^{\circ}$ from the center of the Sun. 


\begin{tabular}{|c|c|c|c|}
\hline \hline Parameter & gaugino & mixed & higgsino \\
\hline \hline $\tan \beta$ & 7.94 & 52.63 & 4.76 \\
\hline$M_{2}(\mathrm{GeV})$ & 148.56 & 457.82 & 3810.98 \\
\hline$M_{3}(\mathrm{GeV})$ & 1847.66 & 2785.12 & 2281.29 \\
\hline$\mu(\mathrm{GeV})$ & 620.95 & 275.72 & 1345.21 \\
\hline$m_{A}(\mathrm{GeV})$ & 1454.49 & 3648.84 & 2716.89 \\
\hline$A_{t}(\mathrm{GeV})$ & 2086.52 & 3607.58 & 5277.32 \\
\hline$A_{\tau}(\mathrm{GeV})$ & -2786.17 & 5256.12 & -4519.05 \\
\hline$m_{\tilde{Q}_{3}}(\mathrm{GeV})$ & 3335.38 & 2330.03 & 3577.35 \\
\hline$m_{\tilde{L}_{3}}(\mathrm{GeV})$ & 144.74 & 1613.40 & 1500.82 \\
\hline \hline$m_{\chi}(\mathrm{GeV})$ & 70.29 & 210.61 & 1360.36 \\
\hline$\sigma v\left(\mathrm{~cm}^{3} \mathrm{~s}^{-1}\right)$ & $1.33 \times 10^{-26}$ & $2.64 \times 10^{-26}$ & $3.75 \times 10^{-25}$ \\
\hline$B_{F}$ & 4.49 & 6.11 & 3.14 \\
\hline \hline
\end{tabular}

Table 3: Our three benchmark p9MSSM DM candidate points, which are denoted by their neutralino compositions. All the three points are consistent with LEP, $\alpha_{T}$, PLANCK, flavor physics, measurements of nuisance parameters, Higgs mass measurements and XENON100 limit at $2 \sigma$.

In Fig. 12(a), we show the $95 \%$ confidence region from the basic+XENON100 profile-likelihood $\left(\Sigma_{\pi N}=43 \pm 12 \mathrm{MeV}\right)$ in the $\left(m_{\chi}, N_{\mu}\right)$ plane, where $N_{\mu}$ is the number of muon events at the detector in 5 years. The color code is the same as in Fig. 6. In Fig. 12](b) we show the case with $\Sigma_{\pi N}=66 \pm 6 \mathrm{MeV}$. One can see from Fig. 12)(a) that many of the points at present not excluded by the XENON100 likelihood with the most conservative choice of theoretical uncertainty, are in reach of the 5 -year sensitivity at IceCube.

In Fig. 12|(c) we show the 95\% confidence region for the basic+XENON100 $\left(\Sigma_{\pi N}=43 \pm\right.$ $12 \mathrm{MeV})$ likelihood in the $\left(m_{\chi}, \sigma_{p}^{\mathrm{SD}}\right)$ plane. The points excluded by the IceCube- 86,5 -year sensitivity are shown as orange crosses. The case with $\Sigma_{\pi N}=66 \pm 6 \mathrm{MeV}$ is shown in Fig. 12.(d).

\subsubsection{Positron flux}

Finally we present the model's predictions for the positron flux from neutralino annihilation in the local halo. Annihilation of DM particles may give rise to a large enough signal of positrons that can be identified by several antimatter search experiments. PAMELA [140] first observed a rise of the cosmic ray positron fraction for positron energies greater than $10 \mathrm{GeV}$. This anomalous enhancement was subsequently confirmed by Fermi-LAT [141] and by the recently released AMS02 first result [142]. In the recent AMS02 result, this continuing rise is extended up to positron energy $\sim 350 \mathrm{GeV}$. Explanation of these anomalies by neutralino DM is not easy, since the described enhancement requires a large boost factor for the annihilation cross section $\sigma v[29$. On the other hand, several groups [143, 144, 145, 146, 147, 148, have fitted the positron results by including AMS02 in a model independent way, and they found that only heavy DM mass, from $m_{\chi} \simeq 800 \mathrm{GeV}$ to a few $\mathrm{TeV}$ can peak at the right position of positron spectrum.

One must bear in mind that in the p9MSSM analyzed in this study, the positron flux would be suppressed by such large masses. However, one can also consider the case in which such enhancement could come from the combined effect of nearby pulsars and DM annihilation, so that $m_{\chi}$ can be lowered down to a few hundred $\mathrm{GeV}$ [146].

Similar to what we did for the $\gamma$-ray flux, we employ the Einasto profile with the caution that 


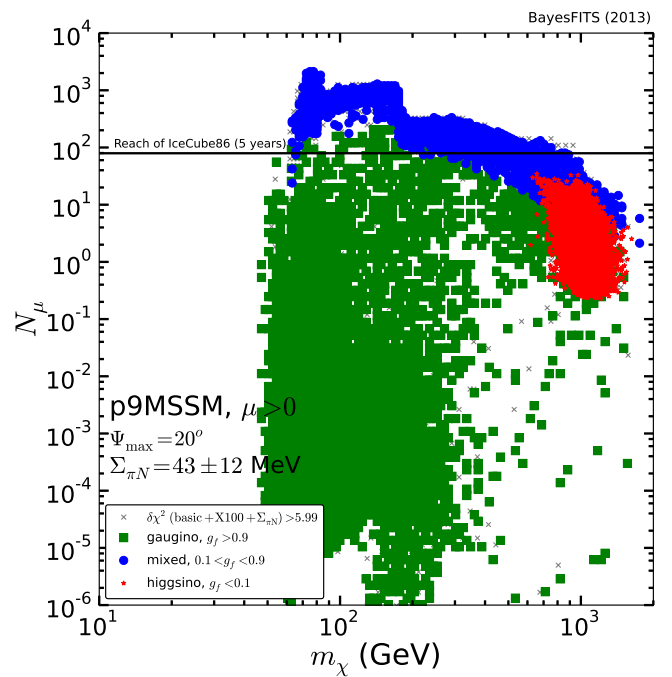

(a)

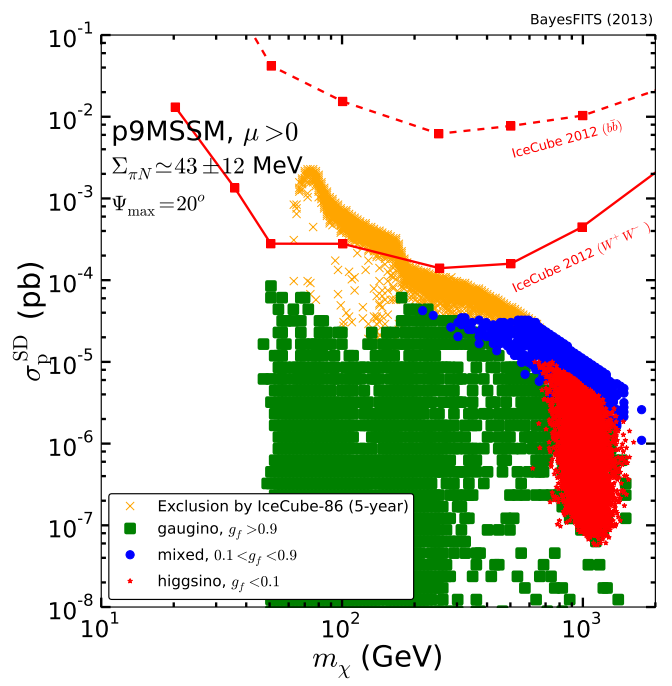

(c)

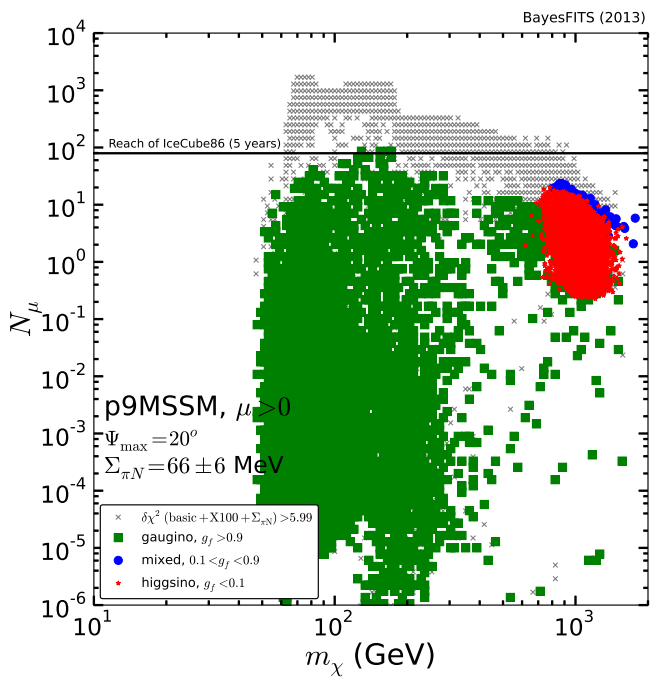

(b)

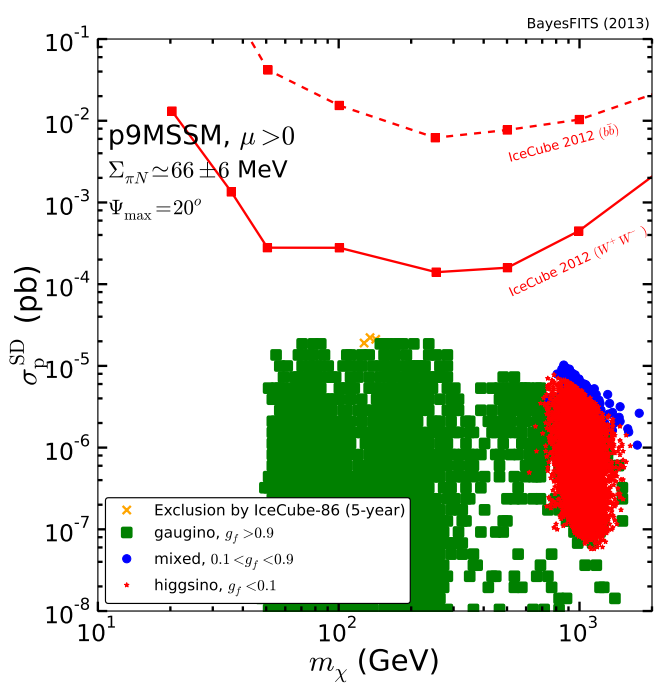

(d)

Figure 12: (a) p9MSSM points allowed at $2 \sigma$ by the basic constraints in the $\left(m_{\chi}, N_{\mu}\right)$ plane. The points consistent at $2 \sigma$ with the basic and XENON100 $\left(\Sigma_{\pi N}=43 \pm 12 \mathrm{MeV}\right)$ constraints are characterized by the composition of the neutralino: gaugino-like (green squares), mixed (blue circles), or higgsino-like (red stars). The expected 95\% C.L. sensitivity of IceCube-86 with 5 years of data is shown with a horizontal solid line. (b) Same as (a), but with $\Sigma_{\pi N}=66 \pm 6 \mathrm{MeV}$. (c) p9MSSM points consistent at $2 \sigma$ with the basic and XENON100 $\left(\Sigma_{\pi N}=43 \pm 12 \mathrm{MeV}\right)$ constraints in the $\left(m_{\chi}, \sigma_{p}^{\mathrm{SD}}\right)$ plane. The points to be excluded by IceCube-86 (5-year sensitivity) are shown as orange crosses. Achieved 95\% C.L. limits from IceCube-79 [139] for neutralinos annihilating into $b \bar{b}$ and $W^{+} W^{-}$channels are shown as dashed red and solid red lines, respectively. Note, however, that for $m_{\chi}<m_{W}$, the solid red line shows the limit for neutralinos annihilating into $\tau^{+} \tau^{-}$. (d) Same as (c), but with $\Sigma_{\pi N}=66 \pm 6 \mathrm{MeV}$. 


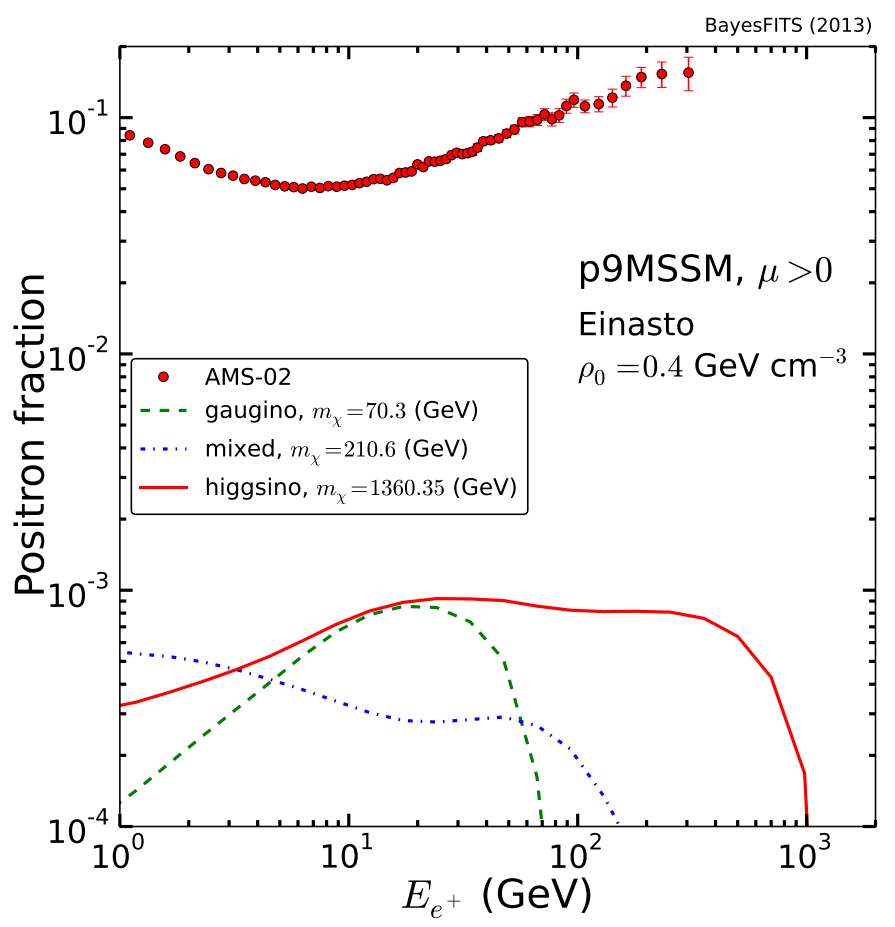

Figure 13: Positron flux fraction from dark matter annihilation in the Galactic halo versus the positron energy $E_{e^{+}}$, for three p9MSSM points with different neutralino composition (shown in Table 3): the point with gaugino-like neutralino is shown with a dashed green line, mixed neutralino with a dash-dotted blue line and higgsino-like neutralino with a solid red line. We assume a boost factor of $B_{F}=1$, a local density of $\rho=0.4 \mathrm{GeVcm}^{-3}$ and the Einasto profile. The AMS-02 data is shown with red circles. The highest part of the positron fraction distributions would reach the AMS-02 data if the boost factors were $B_{F} \geq 65,120$, and 60 for gaugino-like, mixed, and higgsino-like neutralino, respectively.

the halo uncertainties can affect the $\gamma$-ray flux by as much as a factor of 10 [149], but they do not affect the positron flux. This is because positrons will lose energy during the propagation and, since most high-energy positrons originate from a local neighborhood the size of a few kpc [150, 151], their flux is less dependent on the halo. However, for the purpose of this paper here we ignore the uncertainties and we use the best-fit propagation model from the GALPROP group [137, in which they fitted a number of isotopic abundance ratios such as $\mathrm{B} / \mathrm{C}, \mathrm{Be}^{10} / \mathrm{Be}^{9}$ and others. Again, we set the local density to $\rho_{0}=0.4 \mathrm{GeV} \cdot \mathrm{cm}^{-3}$. We modified DarkSUSY to generate the positron-flux spectrum $d N_{e^{+}} / d E_{e^{+}}$. The source term for solving the diffusion equation to obtain the positron spectrum is given in terms of the DM density profile $\rho$ as a function of the distance from the GC, $\mathbf{r}$,

$$
Q_{e^{+}}\left(\mathbf{r}, E_{e^{+}}\right) \sim \frac{\rho(\mathbf{r})^{2}}{m_{\chi}^{2}} \times \sigma v \times \frac{d N_{e^{+}}}{d E_{e^{+}}}
$$

This source term is then fed into GALPROP with the running parameters of the best-fit model [137].

Since we are interested in the positron fraction at large energy, we do not include the solar modulation effect. In Fig. 13, we present the positron flux fraction produced by DM annihilation in the Galactic halo against the positron energy $E_{e^{+}}$for a point with gaugino-like neutralino (blue 
dot-dashed), mixed composition (green dashed) and higgsino-like neutralino (red solid). We find that the neutralino contribution to the positron fraction does not match the observed data. In order to do so, one would require a boost factor of order 65,120 , and 60 for the three cases, respectively.

It is a known fact that $\sigma v$ for $1 \mathrm{TeV}$ higgsino-like (or wino-like, for that matter) neutralinos can be boosted by a Sommerfeld enhancement factor of order 3-6 [152, 153, 154] (for multi-TeV neutralinos the $\sigma v$ boost factor could even be of several orders of magnitude). However, for $m_{\chi} \lesssim$ $1 \mathrm{TeV}$ the Sommerfeld enhancement would still not be enough to obtain the boost factors cited above. On the other hand, the boost factor can also come from astrophysical sources like minispikes [155] or subhalos [156]. Such astrophysical sources can give an energy-dependent boost factor for positron fluxes. (For example, Fig. 5 of Ref. [155] shows enhancements from mini-spikes of order $10^{4}$ at $E_{e^{+}} \simeq 300 \mathrm{GeV}$ for $\sim 1 \mathrm{TeV}$ DM.)

\subsection{Best fits and mass spectrum}

We identify the best-fit points (the points with the smallest total $\chi^{2}$ ) in four cases: the one due to the basic set of constraints (hereafter called just basic); the one obtained by adding the $(g-2)_{\mu}$ constraint to basic; the one obtained by adding XENON100 to basic; and the one obtained by considering all three sets together. The relative breakdown of the contributions from the different constraints is shown for each case in Figs. 14)(a), 14)(b), 14)(c), and 14)(d), respectively.

The corresponding mass spectra for the best-fit points, together with their $68 \%$ and $95 \%$ confidence regions for the cases in question, are shown in Figs. 15.(a), 15.(b), 15.(c), and 15.(d),

For each best-fit point, we also show in Fig. 14 the contribution due to the EW-production likelihood, although we remind the reader that it is not included in the total $\chi^{2}$ computation, but applied separately, for the reasons explained in Sec. 3.1. As a consequence, it always appears in the figures in green (see caption). In the cases where the $(g-2)_{\mu}$ constraint is included, which tend to favor light sleptons, charginos and neutralinos (see Figs. 15(b) and $15(\mathrm{~d})$ ), we have selected the points with the lower $\chi^{2}$ that are not excluded at the $95 \%$ C.L. by direct electroweakino searches at the LHC, i.e., points with $\delta \chi_{\mathrm{EW}}^{2}<5.99$. In particular, our selected points have $\delta \chi_{\mathrm{EW}}^{2} \simeq 4.5$ and $\delta \chi_{\mathrm{EW}}^{2} \simeq 4$, respectively. Note also that when a particular experiment is not constraining for the parameter space its contribution to the $\chi^{2}$ is approximately zero and the corresponding bar does not show in Fig. 14. This is particularly the case of the $\alpha_{T}$ constraint for the best-fit points since, as can be seen in all four panels of Fig. 15, the squark and gluino masses are favored to be well above the present LHC sensitivity.

The four cases in Fig. 14 exhibit similar behavior; only the contributions from $(g-2)_{\mu}$, XENON100 and EW production differ between scenarios. The contributions from EW precision observables, Higgs mass, CMS direct search and relic density are negligible in the total $\chi^{2}$ at the best-fit points, while contributions from $B$-physics observables $B_{u} \rightarrow \tau \nu$ and $\Delta M_{B_{s}}$ are substantial. The discrepancy between the $\mathrm{SM}$ value $\mathrm{BR}\left(B_{u} \rightarrow \tau \nu\right)_{\mathrm{SM}}=8.014 \times 10^{-5}$ and the measured value (Table 2 is known in the literature [157]. XENON100's $\chi^{2}$ is moderate when not constrained by the likelihood (Fig. 14|(a) and Fig. 14|(b)), but when it is included in the likelihood its $\chi^{2}$ is reduced without damaging other predictions (compare Fig. 14)(a) with Fig. 14)(c)).

The $\chi^{2}$ from $(g-2)_{\mu}$ is substantial when not constrained in the likelihood (Fig. 14)(a) and Fig. 14(c) $)$ and even when $(g-2)_{\mu}$ is constrained in the likelihood, its $\chi^{2}$ can only be reduced at the expense of an increase in the EW-production $\chi^{2}$ and, to a lesser extent, XENON100 (Fig. 14|(b) and Fig. $14(\mathrm{~d})$, , thus highlighting the tension between these constraints.

As was shown in Sec. 4.2, however, we identified a fairly broad region of the parameter space in good agreement with all the constraints included here. 


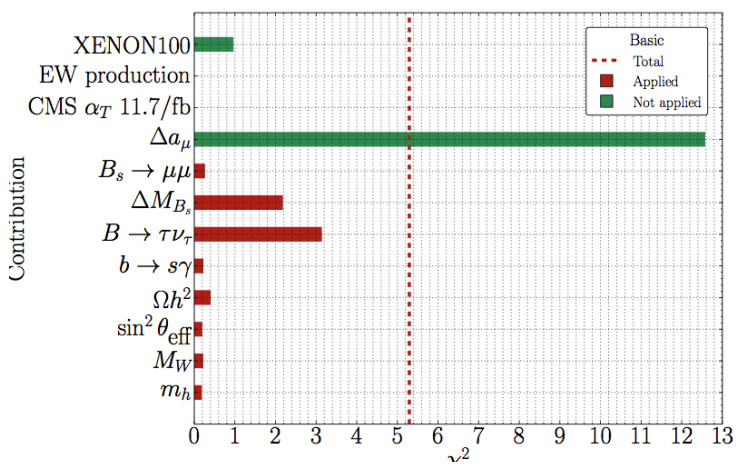

(a) Basic likelihood only

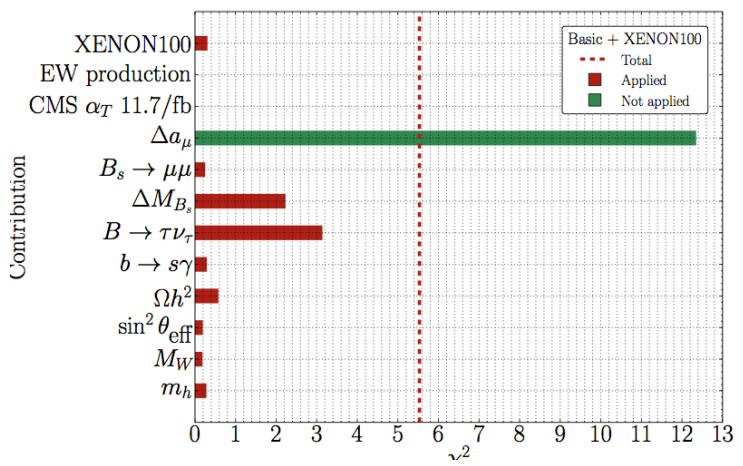

(c) Basic likelihood and XENON100

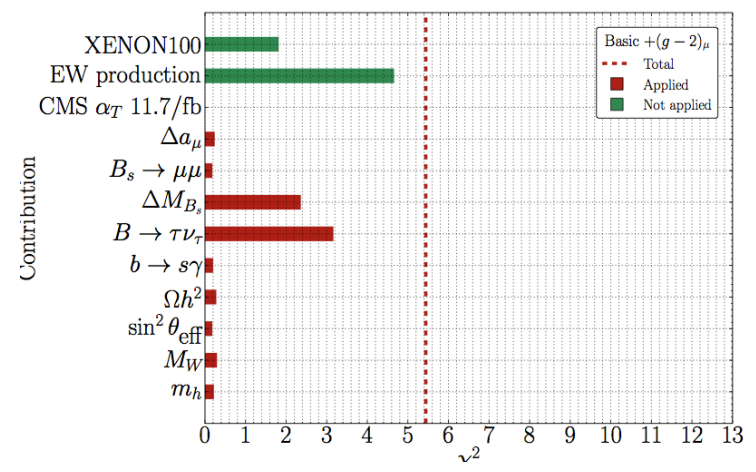

(b) Basic likelihood and $(g-2)_{\mu}$

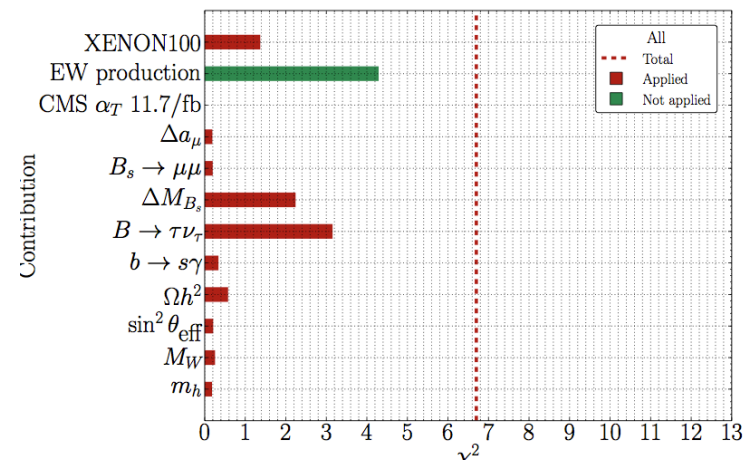

(d) All likelihoods (basic, $(g-2)_{\mu}$ and XENON100)

Figure 14: Breakdown of $\chi^{2}$ at the best-fit points in four likelihood combinations. Note that while in each plot all contributions are plotted, only specific contributions were minimized in each case. Contributions that were (were not) minimized are shown with red, darker (green, lighter) bars. The procedure for identifying these points is described in the text. The total $\chi^{2}$ in each case is marked with a vertical dashed red line.

\section{$5 \quad$ Summary and conclusions}

In this paper we have performed a global statistical analysis of a parametrization of the MSSM with 9 free parameters defined at the SUSY scale, the p9MSSM. The parameters have been selected as a minimum set that allows investigation of the dark matter sector assuming that the lightest neutralino is the only source of dark matter, and that its composition is either bino-like, or higgsinolike, or a mixture of the two. We analyzed a sample of $1.8 \times 10^{6}$ points, which were subject to constraints from the relic density as measured by PLANCK, the Higgs boson mass measurement at the LHC, the recent evidence of $\mathrm{BR}\left(B_{s} \rightarrow \mu^{+} \mu^{-}\right)=3.2_{-1.2}^{+1.5} \times 10^{9}$ at $\mathrm{LHCb}$, the measurement of $\delta(g-2)_{\mu}$, direct SUSY limits from the LHC at $\sqrt{s}=8 \mathrm{TeV}$, and the upper bound on $\sigma_{p}^{\mathrm{SI}}$ from XENON100.

The statistical analysis was performed by constructing a global likelihood function and we presented $95 \%$ confidence regions in 2D projections of the parameter space according to the profilelikelihood method. The global likelihood function was calculated for each scanned point, and it includes an approximate but reasonably accurate implementation of the inclusive search for squarks 


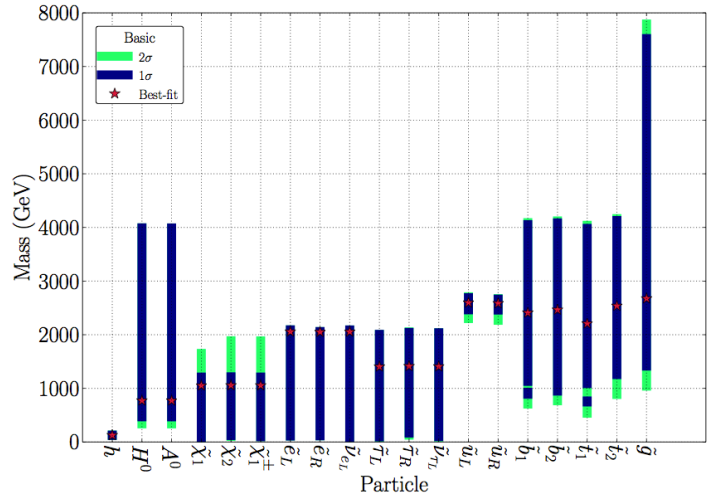

(a) Basic likelihood only

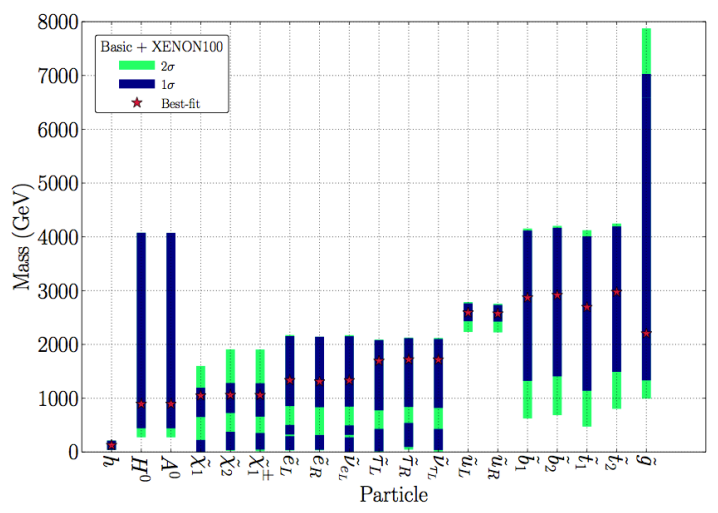

(c) Basic likelihood and XENON100

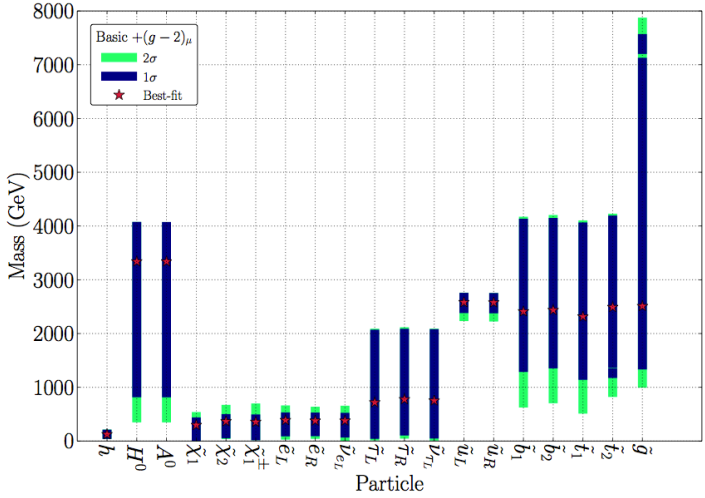

(b) Basic likelihood and $(g-2)_{\mu}$

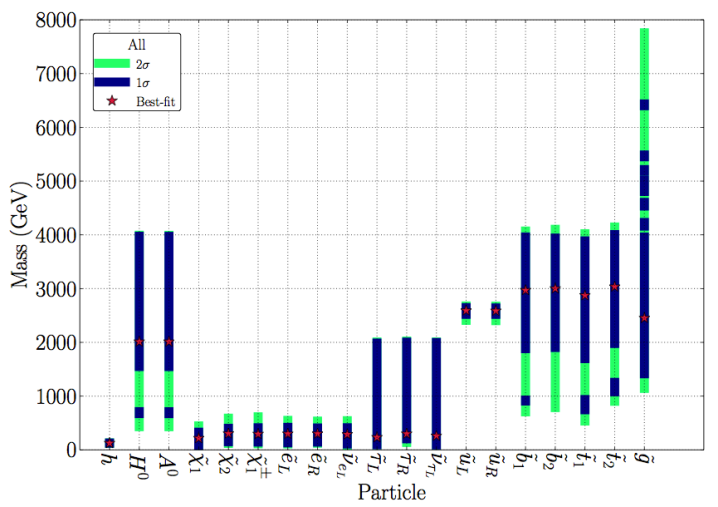

(d) All likelihoods (basic, $(g-2)_{\mu}$ and XENON100)

Figure 15: Best-fitting mass spectra of the p9MSSM in four likelihood combinations (red stars), and the associated $1 \sigma$ (blue, darker bars) and $2 \sigma$ (green, lighter bars) confidence intervals.

and gluinos with the $\alpha_{T}$ variable at $\mathrm{CMS}$, with $11.7 / \mathrm{fb}$ of data at $\sqrt{s}=8 \mathrm{TeV}$. It also includes an approximate but accurate implementation of the XENON100 bound. For the first time we included in the XENON100 likelihood a recent determination [68] from CHAOS data of the $\Sigma_{\pi N}$ term, which parametrizes the effect of the theoretical uncertainties due to nuclear physics on $\sigma_{p}^{\text {SI }}$. We quantified the effect of the new $\Sigma_{\pi N}$ determination on the parameter space and we showed that, when compared to the case where the theoretical uncertainty is neglected, or to cases where the theoretical uncertainty is calculated on the basis of alternative determinations of the same matrix element, the impact of the XENON100 bound is greatly reduced.

In this study, we also analyzed the compatibility of the $(g-2)_{\mu}$ constraint with the strongest lower bounds on the neutralino, chargino, and slepton masses from direct EW-production searches at the LHC. We did so by selecting a uniformly distributed sample of $\sim 2 \%$ of the total points, and by constructing an approximate but accurate likelihood that simulated the CMS $3 l+E_{T}^{\text {miss }}$ search at $\sqrt{s}=8 \mathrm{TeV}$, and $9.2 / \mathrm{fb}$. We found that, when all constraints are taken into account, there is at present a small window in the neutralino mass, $200 \mathrm{GeV} \lesssim m_{\chi} \lesssim 500 \mathrm{GeV}$, and similarly in the sleptons masses, which is not excluded by any one of the constraints, including LHC direct searches and $\delta(g-2)_{\mu}$. The LHC run at $\sqrt{s}=14 \mathrm{TeV}$ should, however, definitely probe this region.

In the last part of the paper, we calculated the impact on the parameter space of the constraints 
on the annihilation cross section $\sigma v$ from Fermi-LAT data on $\gamma$-ray fluxes from dSphs and from the Galactic Center. We confirm the findings of other groups that, in both cases, the present data is constraining for the p9MSSM only when including a phenomenological boost factor. The same conclusion pertains also to the analysis of data from the positron flux at AMS02.

We also considered sensitivities for direct and indirect detection of dark matter for those experiments which will run or collect data in the near future. We showed the projected sensitivities for direct detection at LUX and XENON1T, and for indirect detection we showed the 5-year, 95\% C.L. sensitivity at IceCube- 86 .

Finally, we showed the best-fit points and relative spectra for different combinations of our constraints: basic (for a definition of this box see Sec. 3), basic $+(g-2)_{\mu}$, basic + XENON100, and all combined. The best-fit points show in all four cases squarks an gluinos around $2-3 \mathrm{TeV}$, as required by the Higgs mass measurement and LHC direct searches, with the exception of small regions where the squarks of the third generation are favored around $1 \mathrm{TeV}$, for large stop mixing. The basic set favors sleptons in the ballpark of $1-2 \mathrm{TeV}$, and neutralinos and charginos almost degenerate and around $1 \mathrm{TeV}$, as required in the higgsino region of good relic density. When including the $(g-2)_{\mu}$ constraints the best-fit point shows sleptons, charginos and neutralinos lighter than 500-600 GeV, which is possible for bino, or mixed bino/higgsino LSPs. When including XENON100, but neglecting $(g-2)_{\mu}$, the situation is not dissimilar from the basic case, due the reduced impact of the direct detection constraint with the new determination of $\Sigma_{\pi N}$.

The best-fit $\chi^{2}$ for the case where all contraints are included together is slightly larger than in both the case with basic $+(g-2)_{\mu}$ and the case with basic +XENON100 constraints. This points

to a slight tension between the present constraints from direct detection and the measurement of $\delta(g-2)_{\mu}$.

\section{ACKNOWLEDGMENTS}

We would like to thank Shoaib Munir for helpful discussions through different stages of this work and useful input in the revision of the manuscript. This work has been funded in part by the Welcome Programme of the Foundation for Polish Science. A.J.F. is funded by the Science Technology and Facilities Council (STFC). K.K. is supported by the EU and MSHE Grant No. POIG.02.03.00-00-013/09. L.R. is also supported in part by the Polish National Science Centre Grant No. N202 167440, a STFC consortium grant of Lancaster, Manchester, and Sheffield Universities, and by the EC 6th Framework Programme No. MRTN-CT-2006-035505. The use of the CIS computer cluster at the National Centre for Nuclear Research is gratefully acknowledged. 


\begin{tabular}{|c|c|}
\hline \hline Abbreviation & Full text \\
\hline \hline AF & A-funnel \\
(C)MSSM & (Constrained) Minimal Supersymmetric Standard Model \\
DD & Direct detection \\
DM & Dark matter \\
dSphs & Dwarf spheroidal satellite galaxies \\
EW & Electroweak \\
FP/HB & Focus point/Hyperbolic branch \\
GC & Galactic Center \\
GUT & Grand Unified Theory \\
HR & Higgs-resonance \\
ID & Indirect detection \\
LSP & Lightest supersymmetric particle \\
NUHM & Non-universal Higgs Model \\
p $n$ MSSM & Phenomenological MSSM with $n$ free parameters \\
SC & Slepton coannihilation \\
SI & Spin-independent \\
SM & Standard Model \\
SMS & Simplified model spectrum \\
SUSY & Supersymmetry \\
vev & Vacuum expectation value \\
1TH & TwoV higgsino \\
DD & Two-dimensional \\
\hline \hline
\end{tabular}

Table 4: List of abbreviation acronyms appearing in the text.

\section{A Abbreviations used in the paper}

The abbreviation acronyms appearing in this paper are summarized in Table 4.

\section{B Relation of $\sigma v$ with the relic density}

We dedicate this appendix to reviewing the relationship between the velocity-averaged cross section for annihilation and coannihilation of neutralinos in the early Universe, which enters the calculation of the relic density, and the cross section times velocity in the limit of small momenta $\sigma v$, shown in Fig. 10.

Let us start with $\sigma v$, which is the relevant quantity in indirect DM searches. Since the relative velocities of DM particles are at present very small relative to those in the early Universe at the time of freeze-out, $\sigma v$ is the neutralino pair-annihilation cross section $\sigma v_{\chi \chi}^{\text {ann }}(p)$ in the limit $p \rightarrow 0$. More precisely, its value is determined by averaging over the distribution of small DM velocities in the halo at the present time, and we can thus denote it as $\langle\sigma v\rangle_{\mathrm{ID}}^{\mathrm{ann}}$.

On the other hand, the value of the relic density, $\Omega_{\chi} h^{2}$, is calculated by taking the thermally averaged $\langle\sigma v\rangle_{\text {abund }}^{\text {ann }+c o}$ that results from convolving the total $\sigma v^{\text {ann }+c o}(p)$ due to both annihilation and coannihilation mechanisms, with a Boltzmann distribution $\kappa\left(p, m_{\chi}, T\right)$ over the neutralinos' 
(and coannihilating particles') 3-momenta of magnitude $p$ in the early Universe,

$$
\langle\sigma v\rangle_{\text {abund }}^{\text {ann }+ \text { co }} \sim \int \sigma v^{\mathrm{ann}+\mathrm{co}}(p) \kappa\left(p, m_{\chi}, T\right) d p .
$$

The explicit form of $\kappa\left(p, m_{\chi}, T\right)$ is not particularly illuminating for the discussion, and can be found for instance in Eq. (3.9) and Fig. 1 of Ref. [158].

The correct value of the relic density observed in the Universe, $\Omega_{\chi} h^{2} \simeq 0.1$, corresponds to $\langle\sigma v\rangle_{\text {abund }}^{\text {ann }+ \text { co }} \simeq 3 \times 10^{-26} \mathrm{~cm}^{3} \mathrm{~s}^{-1}$. Note that, on the other hand, $\langle\sigma v\rangle_{\mathrm{ID}}^{\mathrm{ann}}$ is not bound to assume

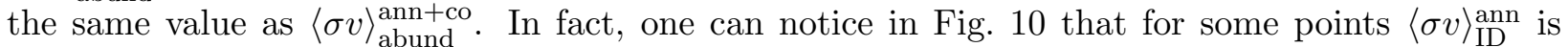
significantly smaller than $3 \times 10^{-26} \mathrm{~cm}^{3} \mathrm{~s}^{-1}$, while for others it is larger. Still, the points in the plot have been selected on the basis of having the correct $\Omega_{\chi} h^{2}$.

This is easy to explain for the regions of the parameter space where early-Universe coannihilation with some particle other than the neutralino is involved, like the SC region or part of the $1 \mathrm{TH}$ region, characterized by $\chi_{1} \chi^{ \pm}$coannihilation and/or $\chi_{1} \chi_{2}$ coannihilation. There, the annihilation cross section does not have to be $\sim 3 \times 10^{-26} \mathrm{~cm}^{3} \mathrm{~s}^{-1}$, because the deficit to fulfil the relic density constraint is made up by the coannihilation cross section.

However, the discrepancy can also be explained for the regions where only neutralino pairannihilation is involved, $\langle\sigma v\rangle_{\text {abund }}^{\text {ann }+ \text { co }} \approx\langle\sigma v\rangle_{\text {abund }}^{\text {ann }}$, due to the effects of thermal averaging. In what follows, we show the effects of Eq. (13) for some benchmark points.

In Fig. 16|(a) we show with a solid thin red line the rescaled annihilation cross section, $\sigma v_{\chi \chi}^{\text {ann }}(p) /\left(10^{-20} \mathrm{~cm}^{3} \mathrm{~s}^{-1}\right)$ (we neglect the suffix $\chi \chi$ in what follows), and we show with a dashed thin black line the rescaled Boltzmann factor, $\kappa\left(p, m_{\chi}, T\right) / \mathrm{GeV}^{-1}$, for a point representative of the $\mathrm{HR}$ region. The corresponding $\langle\sigma v\rangle_{\mathrm{ID}}^{\mathrm{ann}}$ is shown with a dashed thick blue line, and $\langle\sigma v\rangle_{\text {abund }}^{\text {ann }}$ with a solid thick magenta line. One can see that the distribution of $\sigma v^{\text {ann }}(p)$ drops quickly for $p \rightarrow 0$. When the neutralino annihilates at rest the cross section is suppressed by the off-shell propagator. At $p \simeq 25-40 \mathrm{GeV}$ the resonance with $m_{h} \simeq 125 \mathrm{GeV}$ is met (a small peak in $\sigma v^{\text {ann }}(p)$ is observed) and for larger $p$ the cross section keeps increasing thanks to the opening $W W$ pair-production channels. Since the Boltzmann distribution $\kappa\left(p, m_{\chi}, T\right) / \mathrm{GeV}^{-1}$ has a sharp maximum for $p>0$ (typical of a light neutralino mass) the thermal averaging yields $\langle\sigma v\rangle_{\text {abund }}^{\text {ann }} \simeq 10^{-26} \mathrm{~cm}^{3} \mathrm{~s}^{-1}$.

In Fig. 16|(b) we show $\sigma v^{\text {ann }}(p)$ and $\kappa\left(p, m_{\chi}, T\right)$ for a point of the AF region, for which $\langle\sigma v\rangle_{\mathrm{ID}}^{\mathrm{ann}}<$ $\langle\sigma v\rangle_{\text {abund }}^{\text {ann }}$. In this region the correct value of relic density is obtained through the exchange of the pseudoscalar $A$ in the $s$ channel, which requires $s \approx m_{A}^{2}$. Thus, one can see that $\sigma v^{\text {ann }}(p)$ shows a relatively broad peak at $p \simeq 300 \mathrm{GeV}$. The Boltzmann distribution is fairly flat given the large value of the neutralino mass, so that the convolution can produce the correct value of $\langle\sigma v\rangle_{\text {abund }}^{\text {ann }}$.

In Fig. 16(c) we consider a point of the 1 TH region, for which $\left.\langle\sigma v\rangle_{\mathrm{ID}}^{\mathrm{ann}}\right\rangle\langle\sigma v\rangle_{\text {abund }}^{\text {ann }}$. This point is characterized by $m_{A} \approx 2 m_{\chi}$, so that the correct value of the relic density can be obtained, partially at least, again through $A$-resonance in the $s$ channel. With increasing $p$, the resonance region is increasingly left behind, so that $\sigma v^{\text {ann }}(p)$ decreases. The large value of $m_{\chi}$ makes $\kappa\left(p, m_{\chi}, T\right)$ fairly flat over almost all of the momentum range, with the exception of $p \rightarrow 0$, where it is suppressed by $p^{2}$ in the Jacobian. This is enough to bring the convolution down to the correct $\langle\sigma v\rangle_{\text {abund }}^{\text {ann }}$.

Finally, in Fig. 16|(d) we show the results for a point of the FP/HB region, for which $\langle\sigma v\rangle_{\text {abund }}^{\text {ann }}$ and $\langle\sigma v\rangle_{\mathrm{ID}}^{\mathrm{ann}}$ are comparable. As one can see, $\sigma v^{\mathrm{ann}}(p)$ is almost flat, since the rapidly falling cross section for $h$ resonance is enhanced at large $p$ by processes with $t$-channel exchange of stops and/or charginos. The convolution with the Boltzmann factor will thus not change significantly the value of the cross section. 


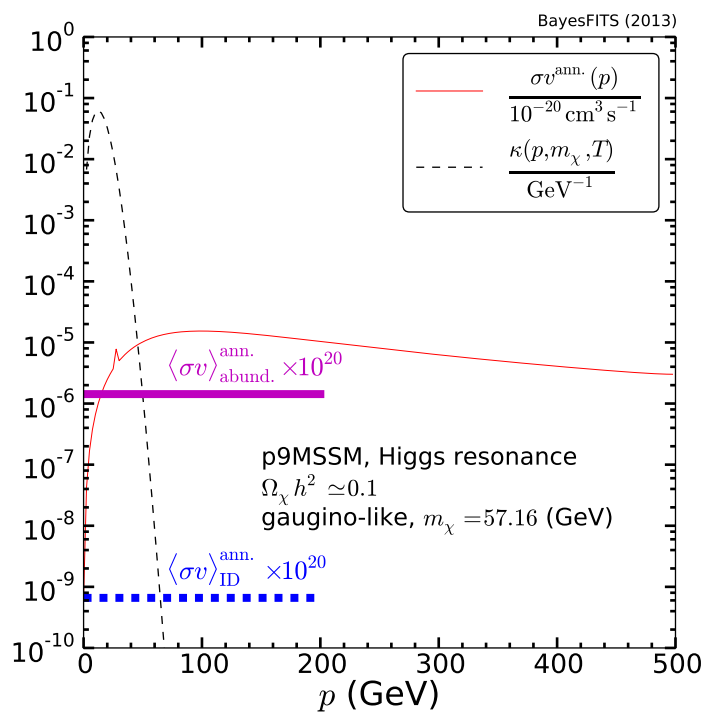

(a)

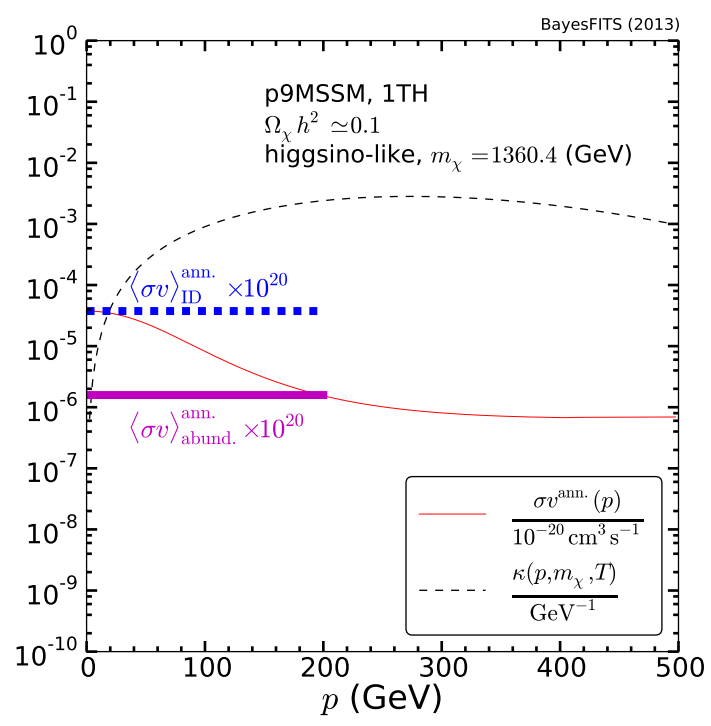

(c)

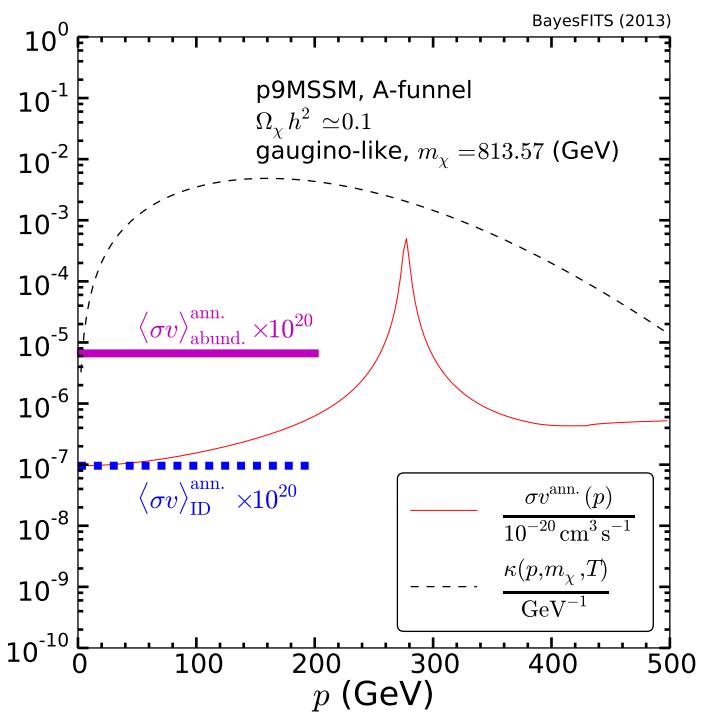

(b)

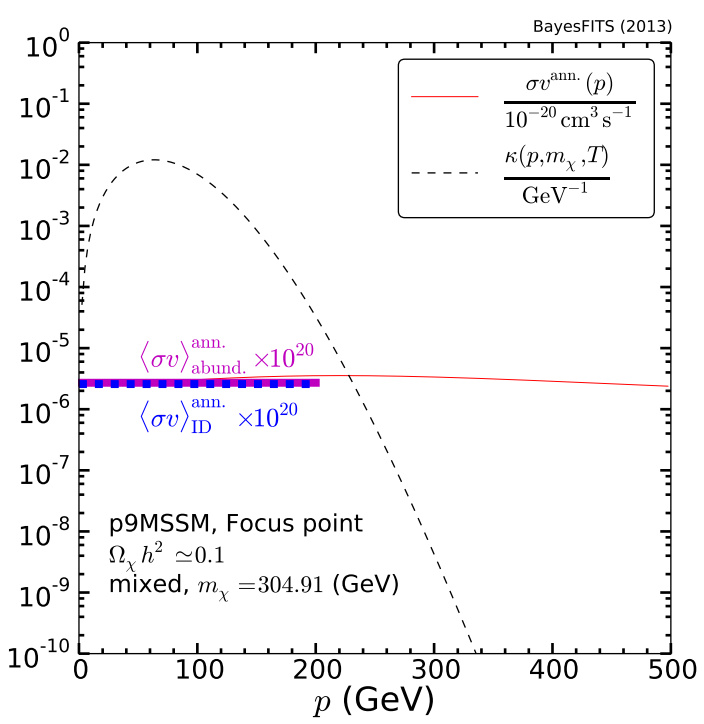

(d)

Figure 16: The Boltzmann factor, $\kappa$ (dashed thin in black), and the annihilation cross section, $\sigma v$ (solid thin in red), versus effective neutralino momentum ( $p=\left|\overrightarrow{p_{2}}\right|=\left|\overrightarrow{p_{1}}\right|$ for annihilating neutralinos labelled $\chi_{1} \chi_{2}$ ) for four p9MSSM points for which the neutralino relic density is achieved via distinct mechanisms: (a) Higgs resonance, (b) $A$-funnel, (c) $\sim 1 \mathrm{TeV}$ higgsino, and (d) the focus point with mixed-composition neutralinos. The thermally averaged cross section, which is

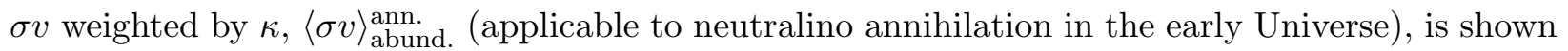
with a solid thick magenta line, while that over low momenta, $\langle\sigma v\rangle_{\mathrm{ID}}^{\mathrm{ann}}$. (applicable to indirect detection), is shown with a dashed thick blue line. Note that while we display only annihilation cross sections, throughout our analysis the relic densities include coannihilation effects, as well. 


\section{References}

[1] CMS Collaboration, S. Chatrchyan et al., "Observation of a new boson at a mass of $125 \mathrm{GeV}$ with the CMS experiment at the LHC," Phys.Lett. B716 (2012) 30-61, arXiv:1207.7235 [hep-ex].; ATLAS Collaboration, G. Aad et al., "Observation of a new particle in the search for the Standard Model Higgs boson with the ATLAS detector at the LHC," Phys.Lett. B716 (2012) 1-29, arXiv:1207.7214 [hep-ex].

[2] "Combination of standard model higgs boson searches and measurements of the properties of the new boson with a mass near 125 gev," Tech. Rep. CMS-PAS-HIG-13-005, CERN, Geneva, 2013

[3] "Combined measurements of the mass and signal strength of the higgs-like boson with the atlas detector using up to $25 \mathrm{fb}^{-1}$ of proton-proton collision data," Tech. Rep. ATLAS-CONF2013-014, CERN, Geneva, Mar, 2013

[4] L. J. Hall, D. Pinner, and J. T. Ruderman, "A Natural SUSY Higgs Near 126 GeV," JHEP 1204 (2012) 131, arXiv:1112.2703 [hep-ph].

[5] H. Baer, V. Barger, and A. Mustafayev, "Implications of a $125 \mathrm{GeV}$ Higgs scalar for LHC SUSY and neutralino dark matter searches," Phys.Rev. D85 (2012) 075010, arXiv:1112.3017 [hep-ph].

[6] S. Heinemeyer, O. Stal, and G. Weiglein, "Interpreting the LHC Higgs Search Results in the MSSM," Phys.Lett. B710 (2012) 201-206, arXiv:1112.3026 [hep-ph].

[7] A. Arbey et al., "Implications of a $125 \mathrm{GeV}$ Higgs for supersymmetric models," Phys.Lett. B708 (2012) 162-169, arXiv:1112.3028 [hep-ph]. ; A. Arbey, M. Battaglia, A. Djouadi, and F. Mahmoudi, "The Higgs sector of the phenomenological MSSM in the light of the Higgs boson discovery," JHEP 1209 (2012) 107, arXiv:1207.1348 [hep-ph].

[8] M. Carena, S. Gori, N. R. Shah, and C. E. Wagner, "A 125 GeV SM-like Higgs in the MSSM and the $\gamma \gamma$ rate," JHEP 1203 (2012) 014, arXiv:1112.3336 [hep-ph].

[9] J. Cao et al., "A SM-like Higgs near $125 \mathrm{GeV}$ in low energy SUSY: a comparative study for MSSM and NMSSM," JHEP 1203 (2012) 086, arXiv:1202.5821 [hep-ph].

[10] N. D. Christensen, T. Han, and S. Su, "MSSM Higgs Bosons at The LHC," Phys.Rev. D85 (2012) 115018, arXiv:1203.3207 [hep-ph].

[11] F. Brummer, S. Kraml, and S. Kulkarni, "Anatomy of maximal stop mixing in the MSSM," JHEP 1208 (2012) 089, arXiv: 1204.5977 [hep-ph].

[12] A. Arbey, M. Battaglia, and F. Mahmoudi, "Light Neutralino Dark Matter in the pMSSM: Implications of LEP, LHC and Dark Matter Searches on SUSY Particle Spectra," Eur.Phys.J. C72 (2012) 2169, arXiv:1205.2557 [hep-ph].

[13] M. Carena, S. Gori, N. R. Shah, C. E. Wagner, and L.-T. Wang, "Light Stau Phenomenology and the Higgs $\gamma \gamma$ Rate," JHEP 1207 (2012) 175, arXiv:1205.5842 [hep-ph].

[14] S. Mohanty, S. Rao, and D. Roy, "Reconciling the muon g - 2 and dark matter relic density with the LHC results in nonuniversal gaugino mass models," JHEP 1309 (2013) 027, arXiv:1303.5830 [hep-ph]. 
[15] T. Han, Z. Liu, and A. Natarajan, "Dark Matter and Higgs Bosons in the MSSM," arXiv:1303.3040 [hep-ph].

[16] J. Ellis and K. A. Olive, "Revisiting the Higgs Mass and Dark Matter in the CMSSM," Eur.Phys.J. C72 (2012) 2005, arXiv:1202.3262 [hep-ph].

[17] H. Baer, V. Barger, and A. Mustafayev, "Neutralino dark matter in mSUGRA/CMSSM with a 125 GeV light Higgs scalar," JHEP 1205 (2012) 091, arXiv:1202.4038 [hep-ph].

[18] P. Bechtle et al., "Constrained Supersymmetry after two years of LHC data: a global view with Fittino," JHEP 1206 (2012) 098, arXiv: 1204.4199 [hep-ph].

[19] C. Balazs et al., "Should we still believe in constrained supersymmetry?," arXiv:1205.1568 [hep-ph].

[20] A. Fowlie, M. Kazana, K. Kowalska, S. Munir, L. Roszkowski, et al., "The CMSSM Favoring New Territories: The Impact of New LHC Limits and a $125 \mathrm{GeV}$ Higgs," Phys.Rev. D86 (2012) 075010, arXiv:1206.0264 [hep-ph].

[21] O. Buchmueller, R. Cavanaugh, M. Citron, A. De Roeck, M. Dolan, et al., "The CMSSM and NUHM1 in Light of 7 TeV LHC, Bs to mu+mu- and XENON100 Data," Eur.Phys.J. C72 (2012) 2243, arXiv:1207.7315 [hep-ph].

[22] S. Akula, P. Nath, and G. Peim, "Implications of the Higgs Boson Discovery for mSUGRA," Phys.Lett. B717 (2012) 188-192, arXiv:1207.1839 [hep-ph].

[23] C. Strege, G. Bertone, F. Feroz, M. Fornasa, R. R. de Austri, et al., "Global Fits of the cMSSM and NUHM including the LHC Higgs discovery and new XENON100 constraints," JCAP 1304 (2013) 013, arXiv:1212.2636 [hep-ph].

[24] M. E. Cabrera, J. A. Casas, and R. R. de Austri, "The health of SUSY after the Higgs discovery and the XENON100 data," arXiv:1212.4821 [hep-ph].

[25] K. Kowalska, L. Roszkowski, and E. M. Sessolo, "Two ultimate tests of constrained supersymmetry," JHEP 1306 (2013) 078, arXiv: 1302.5956 [hep-ph].

[26] G. L. Kane, C. F. Kolda, L. Roszkowski, and J. D. Wells, "Study of constrained minimal supersymmetry," Phys. Rev. D49 (1994) 6173-6210, arXiv:hep-ph/9312272 [hep-ph].

[27] V. Berezinsky, A. Bottino, J. R. Ellis, N. Fornengo, G. Mignola, et al., "Neutralino dark matter in supersymmetric models with nonuniversal scalar mass terms," Astropart.Phys. 5 (1996) 1-26, arXiv:hep-ph/9508249 [hep-ph].

[28] S. Profumo and C. E. Yaguna, "A Statistical analysis of supersymmetric dark matter in the MSSM after WMAP," Phys.Rev. D70 (2004) 095004, arXiv:hep-ph/0407036 [hep-ph].

[29] L. Roszkowski, R. Ruiz de Austri, R. Trotta, Y.-L. S. Tsai, and T. A. Varley, "Global fits of the Non-Universal Higgs Model," Phys.Rev. D83 (2011) 015014, arXiv:0903.1279 [hep-ph].

[30] LHCb Collaboration, R. Aaij et al., "First evidence for the decay $B_{s} \rightarrow \mu^{+} \mu^{-}$," Phys.Rev.Lett. 110 (2013) 021801, arXiv:1211.2674 [hep-ex].

[31] A. J. Buras, J. Girrbach, D. Guadagnoli, and G. Isidori, "On the Standard Model prediction for BR(Bs,d to mu+ mu-)," Eur.Phys.J. C72 (2012) 2172, arXiv:1208.0934 [hep-ph]. 
[32] A. J. Buras, R. Fleischer, J. Girrbach, and R. Knegjens, "Probing New Physics with the $B_{s} \rightarrow \mu+\mu$ - Time-Dependent Rate," JHEP 1307 (2013) 77, arXiv:1303.3820 [hep-ph].

[33] "Search for gluino pair production in final states with missing transverse momentum and at least three b-jets using $12.8 \mathrm{fb}-1$ of pp collisions at sqrt(s) $=8$ tev with the atlas detector.," Tech. Rep. ATLAS-CONF-2012-145, CERN, Geneva, Nov, 2012

[34] "Search for squarks and gluinos with the atlas detector using final states with jets and missing transverse momentum and $5.8 \mathrm{fb}^{-1}$ of $\sqrt{s}=8 \mathrm{tev}$ proton-proton collision data," Tech. Rep. ATLAS-CONF-2012-109, CERN, Geneva, Aug, 2012

[35] "Search for direct top squark pair production in final states with one isolated lepton, jets, and missing transverse momentum in sqrts $=8$, tev $p p$ collisions using $21 \mathrm{fb}^{-1}$ of atlas data," Tech. Rep. ATLAS-CONF-2013-037, CERN, Geneva, Mar, 2013

[36] "Search for direct sbottom production in event with two b-jets using $12.8 \mathrm{fb}-1$ of pp collisions at sqrts $=8$ tev with the atlas detector.," Tech. Rep. ATLAS-CONF-2012-165, CERN, Geneva, Dec, 2012

[37] "Search for top-squark pair production in the single lepton final state in pp collisions at 8 tev," Tech. Rep. CMS-PAS-SUS-13-011, CERN, Geneva, 2013

[38] "Search for direct ewk production of susy particles in multilepton modes with 8tev data," Tech. Rep. CMS-PAS-SUS-12-022, CERN, Geneva, 2012

[39] Muon G-2 Collaboration, G. Bennett et al., "Final Report of the Muon E821 Anomalous Magnetic Moment Measurement at BNL," Phys.Rev. D73 (2006) 072003 , arXiv:hep-ex/0602035 [hep-ex].

[40] M. E. Cabrera, J. A. Casas, R. Ruiz de Austri, and R. Trotta, "Quantifying the tension between the Higgs mass and $(g-2)_{\mu}$ in the CMSSM," Phys.Rev. D84 (2011) 015006 , arXiv:1011.5935 [hep-ph].

[41] M. Cahill-Rowley, J. Hewett, A. Ismail, and T. Rizzo, "SUSY Without Prejudice at the 7 and 8 TeV LHC: Gravitino LSPs," arXiv:1211.7106 [hep-ph].

[42] S. S. AbdusSalam and D. Choudhury, "Higgs boson discovery versus sparticles prediction: Impact on the pMSSM's posterior samples from a Bayesian global fit," arXiv:1210.3331 [hep-ph].

[43] J. A. Conley, J. S. Gainer, J. L. Hewett, M. P. Le, and T. G. Rizzo, "Supersymmetry Without Prejudice at the LHC," Eur.Phys.J. C71 (2011) 1697, arXiv:1009.2539 [hep-ph].

[44] M. W. Cahill-Rowley, J. L. Hewett, S. Hoeche, A. Ismail, and T. G. Rizzo, "The New Look pMSSM with Neutralino and Gravitino LSPs," Eur.Phys.J. C72 (2012) 2156 , arXiv:1206.4321 [hep-ph].

[45] S. S. AbdusSalam, "LHC-7 supersymmetry search interpretation within the pMSSM," Phys.Rev. D87 (2013) 115012, arXiv:1211.0999 [hep-ph]

[46] A. Choudhury and A. Datta, "Neutralino dark matter confronted by the LHC constraints on Electroweak SUSY signals," arXiv:1305.0928 [hep-ph]. 
[47] M. Cahill-Rowley, R. Cotta, A. Drlica-Wagner, S. Funk, J. Hewett, et al., "Complementarity and Searches for Dark Matter in the pMSSM," arXiv:1305.6921 [hep-ph].

[48] U. Haisch and F. Mahmoudi, "MSSM: Cornered and Correlated," JHEP 1301 (2013) 061, arXiv:1210.7806 [hep-ph].

[49] A. Arbey, M. Battaglia, A. Djouadi, and F. Mahmoudi, "An update on the constraints on the phenomenological MSSM from the new LHC Higgs results," Phys.Lett. B720 (2013) 153-160, arXiv:1211.4004 [hep-ph].

[50] F. Mahmoudi, A. Arbey, M. Battaglia, and A. Djouadi, "Implications of LHC Higgs and SUSY searches for MSSM," arXiv:1211.2794 [hep-ph].

[51] F. Mahmoudi, A. Arbey, and M. Battaglia, "Light neutralino dark matter in MSSM," arXiv:1211.2795 [hep-ph].

[52] M. Carena, J. Lykken, S. Sekmen, N. R. Shah, and C. E. Wagner, "The pMSSM Interpretation of LHC Results Using Rernormalization Group Invariants," Phys.Rev. D86 (2012) 075025 , arXiv:1205.5903 [hep-ph].

[53] M. Carena, G. Nardini, M. Quiros, and C. E. Wagner, "MSSM Electroweak Baryogenesis and LHC Data," JHEP 1302 (2013) 001, arXiv:1207.6330 [hep-ph].

[54] C. Boehm, P. S. B. Dev, A. Mazumdar, and E. Pukartas, "Naturalness of Light Neutralino Dark Matter in pMSSM after LHC, XENON100 and Planck Data," JHEP 1306 (2013) 113 arXiv:1303.5386 [hep-ph].

[55] A. Arbey, M. Battaglia, and F. Mahmoudi, "Constraints on the MSSM from the Higgs Sector: A pMSSM Study of Higgs Searches, $B_{s} \rightarrow \mu^{+} \mu^{-}$and Dark Matter Direct Detection," Eur.Phys.J. C72 (2012) 1906, arXiv:1112.3032 [hep-ph].

[56] A. Arbey, M. Battaglia, F. Mahmoudi, and D. Martinez Santos, "Supersymmetry confronts $B_{s}^{0} \rightarrow \mu^{+} \mu^{-}$: Present and future status," Phys.Rev. D87 (2013) 035026, arXiv: 1212.4887 [hep-ph].

[57] F. Mahmoudi, S. Neshatpour, and J. Orloff, "Supersymmetric constraints from $B_{s} \rightarrow \mu^{+} \mu^{-}$ and $B \rightarrow K * \mu^{+} \mu^{-}$observables," JHEP 1208 (2012) 092, arXiv:1205.1845 [hep-ph].

[58] W. Altmannshofer, M. Carena, N. R. Shah, and F. Yu, "Indirect Probes of the MSSM after the Higgs Discovery," JHEP 1301 (2013) 160, arXiv:1211.1976 [hep-ph].

[59] M. Endo, K. Hamaguchi, S. Iwamoto, and T. Yoshinaga, "Muon g-2 vs LHC in Supersymmetric Models," arXiv:1303.4256 [hep-ph].

[60] M. Ibe, T. T. Yanagida, and N. Yokozaki, "Muon g-2 and $125 \mathrm{GeV}$ Higgs in Split-Family Supersymmetry," JHEP 1308 (2013) 067, arXiv:1303.6995 [hep-ph].

[61] M. Arana-Catania, S. Heinemeyer, and M. Herrero, "New Constraints on General Slepton Flavor Mixing," Phys.Rev. D88 (2013) 015026, arXiv:1304.2783 [hep-ph].

[62] H.-B. Zhang, T.-F. Feng, S.-M. Zhao, and T.-J. Gao, "Lepton-flavor violation and $(g-2)_{\mu}$ in the $\mu \nu \mathrm{SSM}, "$ Nucl.Phys. B873 (2013) 300-324, arXiv:1304.6248 [hep-ph]. 
[63] H.-S. Lee and M. Sher, "Dark Two Higgs Doublet Model," Phys.Rev. D87 (2013) 115009 , arXiv:1303.6653 [hep-ph].

[64] Y. Akrami, C. Savage, P. Scott, J. Conrad, and J. Edsjo, "Statistical coverage for supersymmetric parameter estimation: a case study with direct detection of dark matter," JCAP $\mathbf{1 1 0 7}$ (2011) 002, arXiv:1011.4297 [hep-ph].

[65] CMS Collaboration, S. Chatrchyan et al., "Search for supersymmetry in hadronic final states with missing transverse energy using the variables $\alpha_{T}$ and b-quark multiplicity in pp collisions at $\sqrt{s}=8 \mathrm{TeV}$," arXiv:1303.2985 [hep-ex].

[66] XENON100 Collaboration, E. Aprile et al., "Dark Matter Results from 225 Live Days of XENON100 Data," Phys.Rev.Lett. 109 (2012) 181301, arXiv:1207.5988 [astro-ph.C0].

[67] J. Gasser, H. Leutwyler, and M. Sainio, "Sigma term update," Phys.Lett. B253 (1991) 252259 .

[68] J. Stahov, H. Clement, and G. Wagner, "Evaluation of the Pion-Nucleon Sigma Term from CHAOS data," arXiv:1211.1148 [nucl-th].

[69] K. Kowalska, S. Munir, L. Roszkowski, E. M. Sessolo, S. Trojanowski, et al., "The Constrained NMSSM with a 125 GeV Higgs boson - A global analysis," Phys.Rev. D87 (2013) 115010, arXiv:1211.1693 [hep-ph].

[70] S. Munir, L. Roszkowski, and S. Trojanowski, "Di-photon rate enhancement in the NMSSM with nearly degenerate scalar and pseudoscalar Higgs bosons," arXiv:1305.0591 [hep-ph].

[71] R. Cotta, K. Howe, J. Hewett, and T. Rizzo, "Phenomenological minimal supersymmetric standard model dark matter searches on ice," Phys.Rev. D85 (2012) 035017, arXiv:1105.1199 [hep-ph].

[72] N. Arkani-Hamed, A. Delgado, and G. Giudice, "The Well-tempered neutralino," Nucl.Phys. B741 (2006) 108-130, arXiv:hep-ph/0601041 [hep-ph].

[73] G. Bertone, D. Cerdeno, M. Fornasa, L. Pieri, R. Ruiz de Austri, et al., "Complementarity of Indirect and Accelerator Dark Matter Searches," Phys.Rev. D85 (2012) 055014 , arXiv:1111.2607 [astro-ph.HE].

[74] B. Allanach, "SOFTSUSY: a program for calculating supersymmetric spectra," Comput.Phys.Commun. 143 (2002) 305-331, arXiv:hep-ph/0104145 [hep-ph].

[75] F. Feroz, M. Hobson, and M. Bridges, "MultiNest: an efficient and robust Bayesian inference tool for cosmology and particle physics," Mon.Not.Roy.Astron.Soc. 398 (2009) 1601-1614, arXiv:0809.3437 [astro-ph].

[76] A. Arbey and F. Mahmoudi, "SuperIso Relic: A program for calculating relic density and flavor physics observables in Supersymmetry," Comput.Phys.Commun. 176 (2007) 367-382, arXiv:0906.0369 [hep-ph].

[77] G. Belanger, F. Boudjema, A. Pukhov, and A. Semenov, "micrOMEGAs2.0: a program to calculate the relic density of dark matter in a generic model," Comput.Phys.Commun. 181 (2010) 1277-1292, arXiv:hep-ph/0607059 [hep-ph]. 
[78] P. Gondolo et al., "DarkSUSY: Computing supersymmetric dark matter properties numerically," JCAP 0407 (2004) 008, arXiv:astro-ph/0406204 [astro-ph].

[79] M. Frank, T. Hahn, S. Heinemeyer, W. Hollik, H. Rzehak, et al., "The Higgs Boson Masses and Mixings of the Complex MSSM in the Feynman-Diagrammatic Approach," JHEP 0702 (2007) 047, arXiv:hep-ph/0611326 [hep-ph].

[80] G. Degrassi, S. Heinemeyer, W. Hollik, P. Slavich, and G. Weiglein, "Towards high precision predictions for the MSSM Higgs sector," Eur.Phys.J. C28 (2003) 133-143, arXiv:hep-ph/0212020 [hep-ph].

[81] S. Heinemeyer, W. Hollik, and G. Weiglein, "FeynHiggs: A Program for the calculation of the masses of the neutral CP even Higgs bosons in the MSSM," Comput.Phys.Commun. 124 (2000) 76-89, arXiv: hep-ph/9812320 [hep-ph].

[82] S. Heinemeyer, W. Hollik, and G. Weiglein, "The Masses of the neutral CP - even Higgs bosons in the MSSM: Accurate analysis at the two loop level," Eur.Phys.J. C9 (1999) 343366, arXiv:hep-ph/9812472 [hep-ph].

[83] P. Bechtle, O. Brein, S. Heinemeyer, G. Weiglein, and K. E. Williams, "HiggsBounds 2.0.0: Confronting Neutral and Charged Higgs Sector Predictions with Exclusion Bounds from LEP and the Tevatron," Comput.Phys.Commun. 182 (2011) 2605-2631, arXiv:1102.1898 [hep-ph].

[84] A. Fowlie et al., "Bayesian Implications of Current LHC and XENON100 Search Limits for the Constrained MSSM," Phys.Rev. D85 (2012) 075012, arXiv:1111.6098 [hep-ph].

[85] W. A. Rolke, A. M. Lopez, and J. Conrad, "Limits and confidence intervals in the presence of nuisance parameters," Nucl.Instrum.Meth. A551 (2005) 493-503, arXiv:physics/0403059 [physics].

[86] "Combination of standard model higgs boson searches and measurements of the properties of the new boson with a mass near 125 gev," Tech. Rep. CMS-PAS-HIG-12-045, CERN, Geneva, 2012

[87] Planck Collaboration, P. Ade et al., "Planck 2013 results. XVI. Cosmological parameters," arXiv:1303.5076 [astro-ph.CO].

[88] http://www.slac.stanford.edu/xorg/hfag/rare/2012/radll/index.html

[89] Heavy Flavor Averaging Group Collaboration, Y. Amhis et al., "Averages of b-hadron, c-hadron, and tau-lepton properties as of early 2012," arXiv:1207.1158 [hep-ex].

[90] Particle Data Group Collaboration, J. Beringer et al., "Review of Particle Physics (RPP)," Phys.Rev. D86 (2012) 010001.

[91] J. P. Miller, E. de Rafael, and B. L. Roberts, "Muon (g-2): Experiment and theory," Rept.Prog.Phys. 70 (2007) 795, arXiv:hep-ph/0703049 [hep-ph].

[92] R. R. de Austri, R. Trotta, and L. Roszkowski, "A Markov chain Monte Carlo analysis of the CMSSM," JHEP 0605 (2006) 002, arXiv:hep-ph/0602028 [hep-ph].

[93] K. De Bruyn, R. Fleischer, R. Knegjens, P. Koppenburg, M. Merk, et al., "Branching Ratio Measurements of $B_{s}$ Decays," Phys.Rev. D86 (2012) 014027, arXiv:1204.1735 [hep-ph]. 
[94] K. De Bruyn, R. Fleischer, R. Knegjens, P. Koppenburg, M. Merk, et al., "Probing New Physics via the $B_{s}^{0} \rightarrow \mu^{+} \mu^{-}$Effective Lifetime," Phys.Rev.Lett. 109 (2012) 041801, arXiv:1204.1737 [hep-ph].

[95] F. Mahmoudi, private communication.

[96] M. Carena, S. Heinemeyer, O. Stl, C. Wagner, and G. Weiglein, "MSSM Higgs Boson Searches at the LHC: Benchmark Scenarios after the Discovery of a Higgs-like Particle," arXiv:1302.7033 [hep-ph].

[97] S. Scopel, N. Fornengo, and A. Bottino, "Embedding the $125 \mathrm{GeV}$ Higgs boson measured at the LHC in an effective MSSM: possible implications for neutralino dark matter," Phys.Rev. D88 (2013) 023506, arXiv: 1304.5353 [hep-ph].

[98] B. Bhattacherjee, M. Chakraborti, A. Chakraborty, U. Chattopadhyay, D. Das, et al., "Implications of $98 \mathrm{GeV}$ and $125 \mathrm{GeV}$ Higgs scenario in non-decoupling SUSY with updated ATLAS, CMS and PLANCK data," Phys.Rev. D88 (2013) 035011, arXiv:1305.4020 [hep-ph].

[99] T. Sjostrand, S. Mrenna, and P. Z. Skands, "PYTHIA 6.4 Physics and Manual," JHEP 0605 (2006) 026, arXiv:hep-ph/0603175 [hep-ph].

[100] http://physics.ucdavis.edu/ conway/research/software/pgs/pgs4-general.htm

[101] https://twiki.cern.ch/twiki/pub/CMSPublic/PhysicsResultsSUS12028/SUS-12-028_ result.pdf

[102] XENON100 Collaboration, E. Aprile et al., "The XENON100 Dark Matter Experiment," Astropart.Phys. 35 (2012) 573-590, arXiv:1107.2155 [astro-ph.IM].

[103] XENON100 Collaboration, E. Aprile et al., "Likelihood Approach to the First Dark Matter Results from XENON100," Phys. Rev. D84 (2011) 052003, arXiv:1103.0303 [hep-ex].

[104] XENON100 Collaboration, E. Aprile et al., "Dark Matter Results from 100 Live Days of XENON100 Data," Phys. Rev. Lett. 107 (2011) 131302, arXiv:1104.2549 [astro-ph.CO].

[105] G. Jungman, M. Kamionkowski, and K. Griest, "Supersymmetric dark matter," Phys.Rept. 267 (1996) 195-373, arXiv:hep-ph/9506380 [hep-ph].

[106] J. R. Ellis, K. A. Olive, and C. Savage, "Hadronic Uncertainties in the Elastic Scattering of Supersymmetric Dark Matter," Phys.Rev. D77 (2008) 065026, arXiv:0801.3656 [hep-ph].

[107] R. Catena and P. Ullio, "The local dark matter phase-space density and impact on WIMP direct detection," JCAP 1205 (2012) 005, arXiv:1111.3556 [astro-ph.CO].

[108] L. Roszkowski, E. M. Sessolo, and Y.-L. S. Tsai, "Bayesian Implications of Current LHC Supersymmetry and Dark Matter Detection Searches for the Constrained MSSM," Phys.Rev. D86 (2012) 095005, arXiv:1202.1503 [hep-ph].

[109] H. Denz, P. Amaudruz, J. Brack, J. Breitschopf, P. Camerini, et al., "pi+- p differential cross sections at low energies," Phys.Lett. B633 (2006) 209-213, arXiv:nucl-ex/0512006 [nucl-ex]. 
[110] K. Raywood, S. McFarland, P. Amaudruz, G. Smith, and M. Sevior, "The CHAOS second level trigger: A Fast, programmable ECL trigger for a magnetic spectrometer using multiwire proportional chambers," Nucl.Instrum.Meth. A357 (1995) 296-305.

[111] R. L. Workman, R. A. Arndt, W. J. Briscoe, M. W. Paris, and I. I. Strakovsky, "Parameterization dependence of $t$-matrix poles and eigenphases from a fit to $\pi n$ elastic scattering data," Phys. Rev. C 86 (Sep, 2012) 035202, http://link.aps.org/doi/10.1103/PhysRevC.86. 035202

[112] J. Alarcon, J. Martin Camalich, and J. Oller, "The chiral representation of the $\pi N$ scattering amplitude and the pion-nucleon sigma term," Phys.Rev. D85 (2012) 051503 , arXiv:1110.3797 [hep-ph].; J. Alarcon, L. Geng, J. M. Camalich, and J. Oller, "On the strangeness content of the nucleon," arXiv:1209.2870 [hep-ph].

[113] G. E. Hite, W. B. Kaufmann, and R. J. Jacob, "New evaluation of the piNSigma term," Phys.Rev. C71 (2005) 065201.

[114] K. Cheung, Y.-L. S. Tsai, P.-Y. Tseng, T.-C. Yuan, and A. Zee, "Global Study of the Simplest Scalar Phantom Dark Matter Model," JCAP 1210 (2012) 042, arXiv:1207.4930 [hep-ph].

[115] L. Alvarez-Ruso, T. Ledwig, J. M. Camalich, and M. Vicente-Vacas, "The nucleon mass and pion-nucleon sigma term from a chiral analysis of lattice QCD world data," arXiv:1304.0483 [hep-ph].

[116] R. Arndt, W. Briscoe, I. Strakovsky, R. Workman, and M. Pavan, "Dispersion relation constrained partial wave analysis of $\pi \mathrm{N}$ elastic and $\pi N \rightarrow \eta N$ scattering data: The Baryon spectrum," Phys.Rev. C69 (2004) 035213, arXiv:nucl-th/0311089 [nucl-th].

[117] J. R. Ellis, L. Roszkowski, and Z. Lalak, "Higgs effects on the relic supersymmetric particle density," Phys.Lett. B245 (1990) 545-555.

[118] J. R. Ellis, T. Falk, and K. A. Olive, "Neutralino - Stau coannihilation and the cosmological upper limit on the mass of the lightest supersymmetric particle," Phys.Lett. B444 (1998) 367-372, arXiv: hep-ph/9810360 [hep-ph].

[119] K. L. Chan, U. Chattopadhyay, and P. Nath, "Naturalness, weak scale supersymmetry and the prospect for the observation of supersymmetry at the Tevatron and at the LHC," Phys. Rev. D58 (1998) 096004, arXiv:hep-ph/9710473.

[120] J. L. Feng, K. T. Matchev, and T. Moroi, "Focus points and naturalness in supersymmetry," Phys. Rev. D61 (2000) 075005, arXiv:hep-ph/9909334.

[121] M. Drees and M. M. Nojiri, "The Neutralino relic density in minimal $N=1$ supergravity," Phys.Rev. D47 (1993) 376-408, arXiv:hep-ph/9207234 [hep-ph].

[122] M. Drees and M. Nojiri, "Neutralino - nucleon scattering revisited," Phys.Rev. D48 (1993) 3483-3501, arXiv:hep-ph/9307208 [hep-ph].

[123] LUX Collaboration, D. Akerib et al., "The Large Underground Xenon (LUX) Experiment," Nucl.Instrum.Meth. A704 (2013) 111-126, arXiv:1211.3788 [physics.ins-det].

[124] XENON1T Collaboration, E. Aprile, "The XENON1T Dark Matter Search Experiment," arXiv:1206.6288 [astro-ph.IM]. 
[125] J. F. Gunion, Y. Jiang, and S. Kraml, "Could two NMSSM Higgs bosons be present near 125 GeV?," Phys.Rev. D86 (2012) 071702, arXiv:1207.1545 [hep-ph].

[126] LHC Higgs Cross Section Working Group, S. Dittmaier, C. Mariotti, G. Passarino, and R. Tanaka (Eds.), "Handbook of LHC Higgs Cross Sections: 1. Inclusive Observables," CERN-2011-002 (CERN, Geneva, 2011), arXiv:1101.0593 [hep-ph].

[127] S. Dittmaier, S. Dittmaier, C. Mariotti, G. Passarino, R. Tanaka, et al., "Handbook of LHC Higgs Cross Sections: 2. Differential Distributions," arXiv:1201.3084 [hep-ph].

[128] J. A. Casas, J. M. Moreno, K. Rolbiecki, and B. Zaldivar, "Implications of light charginos for Higgs observables, LHC searches and dark matter," arXiv:1305.3274 [hep-ph].

[129] Y.-L. S. Tsai, Q. Yuan, and X. Huang, "A generic method to constrain the dark matter model parameters from Fermi observations of dwarf spheroids," JCAP 1303 (2013) 018, arXiv:1212.3990 [astro-ph.HE].

[130] http://fermi.gsfc.nasa.gov/ssc/data

[131] T. Sjostrand, S. Mrenna, and P. Z. Skands, "A Brief Introduction to PYTHIA 8.1," Comput.Phys.Commun. 178 (2008) 852-867, arXiv:0710.3820 [hep-ph].

[132] M. Bahr, S. Gieseke, M. Gigg, D. Grellscheid, K. Hamilton, et al., "Herwig++ Physics and Manual," Eur.Phys.J. C58 (2008) 639-707, arXiv:0803.0883 [hep-ph].

[133] J. Cembranos, A. de la Cruz-Dombriz, V. Gammaldi, R. Lineros, and A. Maroto, "Reliability of Monte Carlo event generators for gamma ray dark matter searches," arXiv:1305.2124 [hep-ph].

[134] A. Strong, I. Moskalenko, T. Porter, G. Johannesson, E. Orlando, et al., "The GALPROP Cosmic-Ray Propagation Code," arXiv:0907.0559 [astro-ph.HE].

[135] J. Einasto and U. Haud, "Galactic models with massive corona. I - Method. II - Galaxy," Astronomy and Astrophysics (ISSN 0004-6361) 223 (Oct., 1989) 89-106

[136] P. Salucci, F. Nesti, G. Gentile, and C. Martins, "The dark matter density at the Sun's location," Astron.Astrophys. 523 (2010) A83, arXiv:1003.3101 [astro-ph.GA].

[137] R. Trotta, G. Johannesson, I. Moskalenko, T. Porter, R. R. de Austri, et al., "Constraints on cosmic-ray propagation models from a global Bayesian analysis," Astrophys.J. 729 (2011) 106, arXiv:1011.0037 [astro-ph.HE].

[138] Fermi-LAT Collaboration, "Fermi-LAT Observations of the Diffuse Gamma-Ray Emission: Implications for Cosmic Rays and the Interstellar Medium," Astrophys.J. 750 (2012) 3, arXiv:1202.4039 [astro-ph.HE]

[139] IceCube Collaboration, M. Aartsen et al., "Search for dark matter annihilations in the Sun with the 79-string IceCube detector," Phys.Rev.Lett. 110 (2013) 131302, arXiv:1212.4097 [astro-ph.HE].

[140] PAMELA Collaboration, O. Adriani et al., "PAMELA results on the cosmic-ray antiproton flux from $60 \mathrm{MeV}$ to $180 \mathrm{GeV}$ in kinetic energy," Phys.Rev.Lett. 105 (2010) 121101, arXiv: 1007.0821 [astro-ph.HE] 
[141] The Fermi LAT Collaboration, A. A. Abdo et al., "Measurement of the Cosmic Ray e+ plus e- spectrum from $20 \mathrm{GeV}$ to $1 \mathrm{TeV}$ with the Fermi Large Area Telescope," Phys.Rev.Lett. 102 (2009) 181101, arXiv:0905.0025 [astro-ph.HE]

[142] AMS Collaboration, M. Aguilar et al., "First Result from the Alpha Magnetic Spectrometer on the International Space Station: Precision Measurement of the Positron Fraction in Primary Cosmic Rays of 0.5350 GeV," Phys.Rev.Lett. 110 no. 14, (2013) 141102.

[143] M. Cirelli, M. Kadastik, M. Raidal, and A. Strumia, "Model-independent implications of the e+-, anti-proton cosmic ray spectra on properties of Dark Matter," Nucl.Phys. B813 (2009) 1-21, arXiv:0809.2409 [hep-ph].

[144] J. Kopp, "Constraints on dark matter annihilation from AMS-02 results," arXiv:1304.1184 [hep-ph].

[145] A. De Simone, A. Riotto, and W. Xue, "Interpretation of AMS-02 Results: Correlations among Dark Matter Signals," JCAP 1305 (2013) 003, arXiv:1304.1336 [hep-ph].

[146] Q. Yuan, X.-J. Bi, G.-M. Chen, Y.-Q. Guo, S.-J. Lin, et al., "Implications of the AMS-02 positron fraction in cosmic rays," arXiv:1304.1482 [astro-ph.HE].

[147] I. Cholis and D. Hooper, "Dark Matter and Pulsar Origins of the Rising Cosmic Ray Positron Fraction in Light of New Data From AMS," Phys.Rev. D88 (2013) 023013, arXiv: 1304.1840 [astro-ph.HE].

[148] Q. Yuan and X.-J. Bi, "Reconcile the AMS-02 positron fraction and Fermi-LAT/HESS total $e^{ \pm}$spectra by the primary electron spectrum hardening," arXiv:1304.2687 [astro-ph.HE]

[149] D. T. Cumberbatch, Y.-L. S. Tsai, and L. Roszkowski, "The impact of propagation uncertainties on the potential Dark Matter contribution to the Fermi LAT mid-latitude gamma-ray data," Phys.Rev. D82 (2010) 103521, arXiv:1003.2808 [astro-ph.HE].

[150] E. A. Baltz and J. Edsjo, "Positron propagation and fluxes from neutralino annihilation in the halo," Phys.Rev. D59 (1998) 023511, arXiv:astro-ph/9808243 [astro-ph].

[151] J. Lavalle, J. Pochon, P. Salati, and R. Taillet, "Clumpiness of dark matter and positron annihilation signal: computing the odds of the galactic lottery," Astron.Astrophys. 462 (2007) 827-848, arXiv:astro-ph/0603796 [astro-ph].

[152] M. Cirelli, A. Strumia, and M. Tamburini, "Cosmology and Astrophysics of Minimal Dark Matter," Nucl.Phys. B787 (2007) 152-175, arXiv:0706.4071 [hep-ph].

[153] J. Hisano, S. Matsumoto, M. M. Nojiri, and O. Saito, "Non-perturbative effect on dark matter annihilation and gamma ray signature from galactic center," Phys.Rev. D71 (2005) 063528, arXiv:hep-ph/0412403 [hep-ph]

[154] A. Hryczuk, R. Iengo, and P. Ullio, "Relic densities including Sommerfeld enhancements in the MSSM," JHEP 1103 (2011) 069, arXiv:1010.2172 [hep-ph].

[155] P. Brun, G. Bertone, J. Lavalle, P. Salati, and R. Taillet, "Antiproton and Positron Signal Enhancement in Dark Matter Mini-Spikes Scenarios," Phys.Rev. D76 (2007) 083506, arXiv:0704.2543 [astro-ph]. 
[156] J. Lavalle, Q. Yuan, D. Maurin, and X. Bi, "Full Calculation of Clumpiness Boost factors for Antimatter Cosmic Rays in the light of Lambda-CDM N-body simulation results. Abandoning hope in clumpiness enhancement?," Astron.Astrophys. 479 (2008) 427-452, arXiv:0709.3634 [astro-ph].

[157] T. Hurth and F. Mahmoudi, "New physics search with flavour in the LHC era," Rev.Mod.Phys. 85 (2013) 795, arXiv:1211.6453 [hep-ph].

[158] J. Edsjo, M. Schelke, P. Ullio, and P. Gondolo, "Accurate relic densities with neutralino, chargino and sfermion coannihilations in mSUGRA," JCAP 0304 (2003) 001, arXiv:hep-ph/0301106 [hep-ph]. 